\title{
Improvements in the Resistance of the Banana Species to Fusarium Wilt: A Systematic Review of Methods and Perspectives
}

\author{
Anelita de Jesus Rocha ${ }^{1,+} \mathbb{D}^{D}$, Julianna Matos da Silva Soares ${ }^{1,+}{ }^{\mathbb{D}}$, Fernanda dos Santos Nascimento ${ }^{1(\mathbb{D})}$, \\ Adriadna Souza Santos ${ }^{2}$, , Vanusia Batista de Oliveira Amorim ${ }^{3}$, Claudia Fortes Ferreira ${ }^{3}$, , Fernando Haddad $^{3}$, \\ Janay Almeida dos Santos-Serejo ${ }^{3}$ (D) and Edson Perito Amorim ${ }^{3, *}$ \\ 1 Department of Biological Sciences, State University of Feira de Santana, \\ Feira de Santana 44036-900, Bahia, Brazil; anelitarocha@gmail.com (A.d.J.R.); \\ juliannamatos91@gmail.com (J.M.d.S.S.); feel.20@hotmail.com (F.d.S.N.) \\ 2 Bahia Education Secretary, Salvador 41745-004, Bahia, Brazil; adriadna_souza@yahoo.com.br \\ 3 Embrapa Cassava and Fruit, Cruz das Almas 44380-000, Bahia, Brazil; \\ vanusiaamorim50@gmail.com (V.B.d.O.A.); claudia.ferreira@embrapa.br (C.F.F.); \\ fernando.haddad@embrapa.br (F.H.); janay.serejo@embrapa.br (J.A.d.S.-S.) \\ * Correspondence: edson.amorim@embrapa.br; Tel.: +55-75-3312-8058; Fax: + 55-75-3312-8097 \\ + Authors contributed equally to this manuscript.
}

Citation: Rocha, A.d.J.; Soares, J.M.d.S.; Nascimento, F.d.S.; Santos, A.S.; Amorim, V.B.d.O.; Ferreira, C.F.; Haddad, F.; Santos-Serejo, J.A.d.; Amorim, E.P. Improvements in the Resistance of the Banana Species to Fusarium Wilt: A Systematic Review of Methods and Perspectives. J. Fungi 2021, 7, 249. https://doi.org/ $10.3390 /$ jof7040249

Academic Editors: Guy Blomme, George Mahuku and Miguel Dita

Received: 3 January 2021

Accepted: 22 March 2021

Published: 25 March 2021

Publisher's Note: MDPI stays neutral with regard to jurisdictional claims in published maps and institutional affiliations.

Copyright: (c) 2021 by the authors. Licensee MDPI, Basel, Switzerland. This article is an open access article distributed under the terms and conditions of the Creative Commons Attribution (CC BY) license (https:// creativecommons.org/licenses/by/ $4.0 /)$.
Abstract: The fungus Fusarium oxysporum f. sp. cubense (FOC), tropical race 4 (TR4), causes Fusarium wilt of banana, a pandemic that has threatened the cultivation and export trade of this fruit. This article presents the first systematic review of studies conducted in the last 10 years on the resistance of Musa spp. to Fusarium wilt. We evaluated articles deposited in different academic databases, using a standardized search string and predefined inclusion and exclusion criteria. We note that the information on the sequencing of the Musa sp. genome is certainly a source for obtaining resistant cultivars, mainly by evaluating the banana transcriptome data after infection with FOC. We also showed that there are sources of resistance to FOC race 1 (R1) and FOC TR4 in banana germplasms and that these data are the basis for obtaining resistant cultivars, although the published data are still scarce. In contrast, the transgenics approach has been adopted frequently. We propose harmonizing methods and protocols to facilitate the comparison of information obtained in different research centers and efforts based on global cooperation to cope with the disease. Thus, we offer here a contribution that may facilitate and direct research towards the production of banana resistant to FOC.

Keywords: Musa spp.; Fusarium oxysporum f. sp. cubense; genetic improvement; resistance; state-ofthe-art

\section{Introduction}

Dessert bananas and plantains are very popular fruits worldwide. In 2018, approximately 116 million tons of bananas and 40 million tons of plantains were produced [1]. In terms of exports, bananas are among the most traded fruits globally, with almost 23 million tons (except for plantains) exported in 2017, representing almost $20 \%$ of global production [1]. Approximately 11.3 million hectares are dedicated to banana and plantain production worldwide, and there are more than 1000 varieties produced and consumed locally [2]. The Cavendish banana, which accounts for about $47 \%$ of global production, is the most traded [2]. In African regions, plantains comprise a significant and essential component, contributing considerably to food security and income generation for more than 70 million Africans [3-5]. Similarly, in Latin America and the Caribbean, 62\% of total banana and plantain production (20 million tons) is consumed locally, and approximately 
6.8 million tons of plantains are produced, of which $72 \%$ are traded on international markets, indicating the enormous importance of these crops for local food and food security throughout the region $[6,7]$.

Among banana improvement programs' objectives are achieving cultivars resistant to abiotic stressors, such as salinity [8,9] and drought [10-12]. Another major challenge for the global production of Musaceae species is the development of cultivars resistant to biotic stressors, represented by their primary pests, the banana root borer (Cosmopolites sordidus) and the nematodes Meloidogyne spp., Pratylenchus coffeae, and Radopholus similis [13-19], and disease-causing pathogens, including banana bunchy top virus (BBTV) [20,21], Xanthomonas vasicola pv. musacearum causing bacterial wilt [22-25], Pseudocercospora fijiensis causing black Sigatoka [26-29], and Fusarium oxysporum f. sp. cubense (FOC) causing Fusarium wilt [30-33].

FOC is one of the main biotic stress factors affecting bananas and Fusarium wilt is considered the most destructive and widely spread disease in the banana-producing regions around the world $[34,35]$. The causal agent is a soilborne fungus apparently considered hemibiotrophic; therefore, it initially establishes in a biotrophic relationship interacting with live plant cells of the host, and then in its necrotrophic phase, the host's tissues are dead [30]. Frequently FOC persists in cultivated areas for years due to it is survival phase when it then interacts as saprophytic in cultural remains or produces resting spores known as chlamydospores besides surviving and multiplying in alternative hosts $[30,35,36]$. The disease is characterized by yellowing of the young leaves, and pseudostem splitting, and eventually death of the plant $[30,37,38]$.

Fusarium wilt epidemics caused by race 1 (FOC R1), which occurred in Central America, caused the devastation of the susceptible "Gros Michel" cultivar plantations and was one of the most severe in the history of the crop in the Americas. For this reason, Gros Michel was replaced by cultivars of the subgroup Cavendish that are resistant to FOC R1 [39-41]. However, in the late 1980s, a highly virulent strain of FOC-infected Cavendish cultivars and spread to Asia, Africa, Indonesia, and more recently to South America [30,41,42]. Currently, Fusarium wilt can be considered a pandemic disease because of the spread of the tropical race 4 (FOC TR4) strain [43,44].

Chemical control is unfeasible and minimally effective, and it can be harmful to human health and the environment. Although still in its initial stages, biological control demonstrates promising results $[31,45]$. Low efficacy of the biological control is attributed to inherent factors to the dynamics of the disease's primary inoculum, especially production of chlamydospores, which persists in cultivated areas, such as the capacity to survive in crop remains as an endophytic fungus in alternative hosts $[30,36,46]$. In addition, the genetic variability of the pathogen, resulting in new strains capable of infecting resistant cultivars, is another factor that limits the use of methods of disease management and control [47-49]. Therefore, efforts on the genetic improvement to achieve resistance to FOC R1 and FOC TR4 have been focused on finding resistant cultivars through traditional methods of germplasm selection or the generation of new cultivars by hybridization, genetic transformation, somaclonal variation, or mutation induction [31,50,51].

Until now, there are reviews available in the literature about Fusarium wilt related to epidemiology and disease management [30,35,41,52-54], biological control [45,55], genetic breeding for resistance [56,57] and one review about genomic aspects of Musa spp. for stress resistance [58].

The systematic reviews were mainly developed because of the need for rapid responses to human health issues, and nowadays, this tool has contributed to several study areas [59-61]. However, to our best knowledge, no systematic reviews on the genetic improvement of Musa spp. to resist Fusarium wilt have been published; only studies related to water stress in Musa spp. [62] and banana consumption [63]. Therefore, to provide detailed information on the subject and to collaborate with the information gathered so far, we propose a systematic approach to the studies on Musa spp., with a focus on genetic improvement for resistance to the FOC pathogen, through a systematic review of studies conducted over the last 10 years. 


\section{Materials and Methods}

The free software State of the Art by Systematic Review (StArt) v.3.3 beta 03, developed by the Federal University of São Carlos (UFSCar), was used to perform a systematic review. This tool offers systematized answers to questions directed toward the objective of the review. The review process was performed in three stages-planning, execution, and summarization-according to the review flowchart in Figure 1, which followed the model proposed by Santos et al. [62].

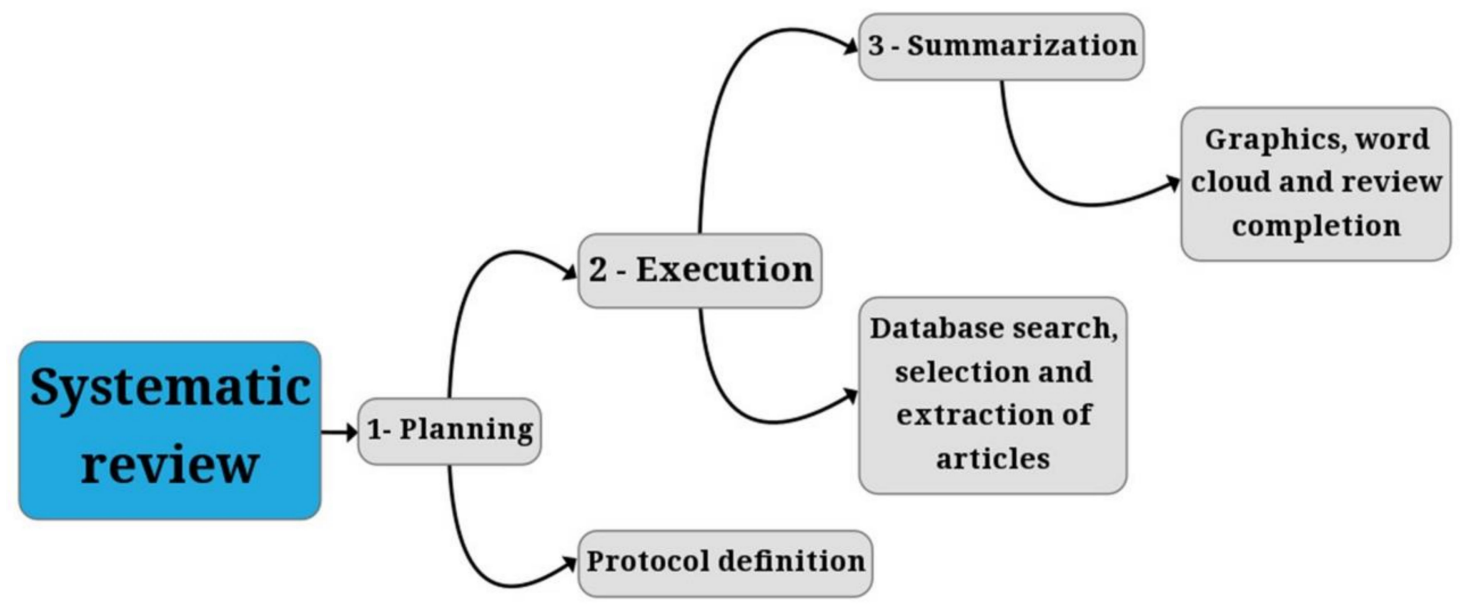

Figure 1. General systematic literature review flowchart. Source: author's compilation.

\subsection{Planning}

A protocol to be followed during the review process was formulated, in which the title, objective, keywords, research questions, research sources, and inclusion/exclusion criteria of articles were defined during their selection and extraction. The StArt protocol is available for download at https://doi.org/10.5281/zenodo.4555385 (accessed on 22 February 2021). The research questions of the review are listed in Table 1.

Table 1. List of questions about the genetic improvement of Musa for resistance to Fusarium wilt to be answered by a systematic review of studies carried out in the last ten years.

\section{Research Questions}

Q1: What are the known sources of resistance (germplasm) to Fusarium oxysporum f. sp. cubense?

Q2: Which breeding programs work on the resistance to Fusarium wilt with respect to cultivar development?

Q3: Which genes are reported associated with resistance to Fusarium oxysporum f. sp. cubense in Musa spp.

Q4: What breeding techniques are associated with overcome Fusarium wilt?

Q5: Which biotechnological tools are used for assisted selection for resistance to Fusarium oxysporum f. sp. cubense?

Q6: Which germplasm collections have information with the potential for genetic improvement to Fusarium wilt?

Q7: What is the frequency of studies by country, and which programs of improvement work with crossbreeding in order to develop resistant cultivars?

Q8: Are there scales to assess the disease? What is the difference between them?

Q9: How often is the banana genome used?

To answer question 7 , when there was no mention in the text of the location where the study was conducted, the search criteria within the article were standardized to the corresponding author's mailing address to obtain information from which country the studies originated. 


\subsection{Execution: Search}

Electronic surveys were conducted on the following databases, aiming to identify publications made available between January 2010 and December 2020: Scopus (http: / / www.scopus.com/), Web of Science (http:/ / apps.isiknowledge.com), PubMed Central (https:/ / www.ncbi.nlm.nih.gov/pmc/about/intro/), Springer (https://www.springer. $\mathrm{com} / \mathrm{br}$ ); Coordination for the Improvement of Higher Education Personnel Portal Journal (http:/ / www.periodicos.capes.gov.br/), and Google Scholar (https:/ / scholar.google.com. $\mathrm{br} / \mathrm{schhp}$ ?hl=en\&as_sdt=0,5), using a standardized search string with the following keywords: Musa spp. and bananas and plantains and Fusarium wilt or Fusarium oxysporum f. sp. cubense or Panama disease and genetic resistance and markers and genes. This set of terms was used for research in all fields within the articles. The Boolean operators AND and OR were used to differentiate the search terms. Search results in each base were imported into BIBTEX, MEDLINE, or RIS formats, compatible with StArt. Relevant documents not found or published after the selection stage started were added manually. We did not consider using the name Fusarium odoratissimum proposed by Maryani et al. [64] in our standardized search due to the low number of published articles using this new suggested nomenclature, and this would limit the number of recovered articles in the database.

\subsection{Execution: Selection and Extraction}

In the selection stage, the articles that contained the terms adopted in the search string in the title, abstract, or keywords were accepted. In the extraction stage, where the number of articles was restricted, a single criterion to include articles was adopted, as follows: (I) articles that answer the protocol's questions (Table 1). The criteria used to exclude articles in the extraction stage were (E) review articles, (E) theses, dissertations, manuals, and book chapters, (E) articles outside the subject, (E) articles published in event annals, (E) articles on genetic diversity of FOC, (E) articles on disease management strategies, and (E) articles on first reports of FOC. These criteria were considered to restrict the selected articles to the focus of this review since they do not answer the proposed questions about improving the resistance of Musa spp. to FOC. The preferred reporting items for systematic reviews and meta-analyses (PRISMA) checklist is presented for download at https:/ / doi.org/10.5281/zenodo.4313617 (accessed on 9 December 2020).

\subsection{Analysis of the Articles}

The process of analyzing the articles was based on the calculation of the frequencies of articles related to each of the research questions. Subsequently, graphs, word clouds, and tables were prepared.

\section{Results}

\subsection{Screening of Studies}

The article screening process is represented by the flow chart in Figure 2. PubMed Central contributed the largest number of articles to this review, with $806(50 \%)$ of the total, followed by Web of Science with 361 (22\%) and Google Scholar with $319(20 \%)$. The other databases, namely Scopus, Springer, and Coordination for the Improvement of Higher Education Personnel Portal Journal, contributed $69(4 \%), 26(2 \%)$, and $8(0.5 \%)$ articles, respectively. Moreover, $22(1.2 \%)$ articles that were not obtained automatically were added manually (Figure 2). 


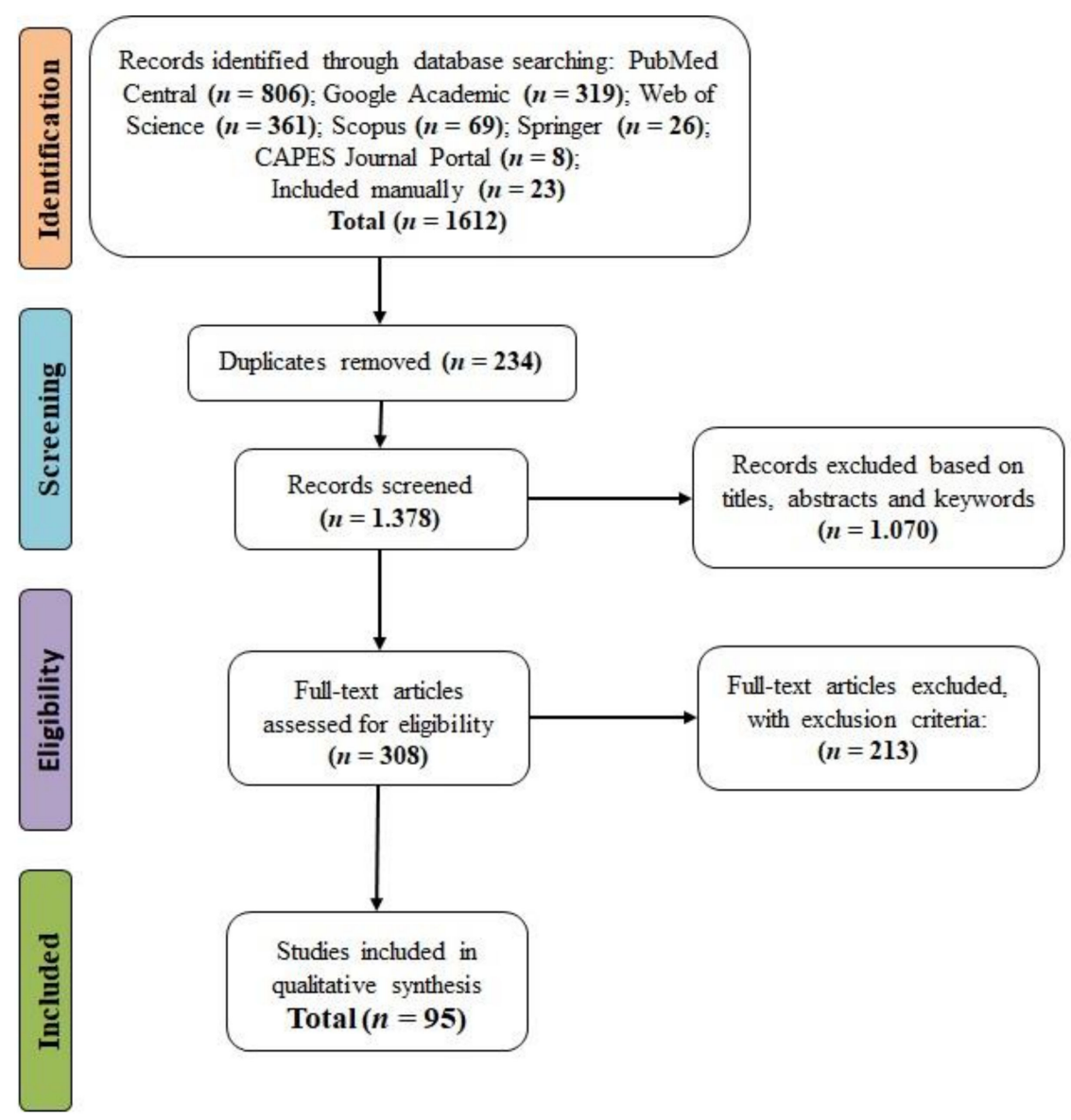

Figure 2. Flow diagram (preferred reporting items for systematic reviews and meta-analyses (PRISMA)). The selection process of studies for inclusion or exclusion in the systematic review on genetic improvement of banana for resistance to Fusarium wilt. $n=$ number of studies. The flow diagram was based on a model available at http:/ / prisma-statement.org/PRISMAStatement/FlowDiagram. CAPES: Coordination for the Improvement of Higher Education Personnel (accessed on 9 December 2020).

We identified 1612 articles from the database tracking, of which 234 were duplicated, and 1377 were eliminated in the selection process by reading the title, abstracts, and keywords, which did not fit the purposes of the research. In the extraction stage, 308 articles were analyzed. After reading the articles entirely, 213 were eliminated; hence, 95 were selected to compose the systematic review (Figure 2). The articles selected to compose the systematic review are available for consultation and download at https:// doi.org/10.528 1/zenodo.4555343 (accessed on 22 February 2021) and its origin and database in Table S2.

A word cloud was generated from the keywords of the 95 articles for this review. As expected, there was a predominance of the articles with the keywords, "Fusarium oxysporum f. sp. cubense", "Fusarium wilt", "Musa", "banana", "disease", "resistance", "race", tropical, and TR4 (Figure 3). Other keywords that had a remarkable frequency in the word cloud were "gene", "Panama", "transformation or transgenesis", "plant", "infection, "green fluorescent protein (GFP)", "protein", "SCAR", "Acuminata", "species", "Cavendish", and "polymerase chain reaction (PCR)" (Figure 3). 


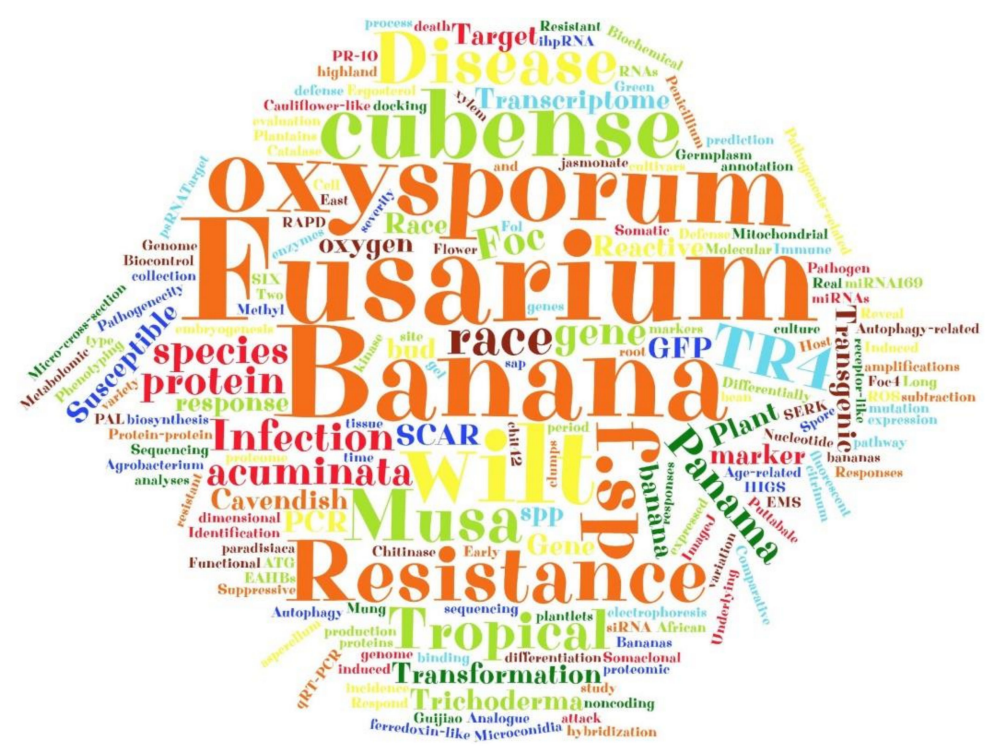

Figure 3. Word cloud generated from article keywords of selected articles to compose a systematic review on breeding Musa to Fusarium wilt. The word cloud was created in a free online generator (https: / / www.wordclouds.com/, accessed on 16 August 2020), based on the frequency of each keyword.

\subsection{Known Origin Sites}

Among the 95 articles, 53\% were from China, followed by India (15\%), Australia $(12 \%)$, Brazil (7\%), Malaysia (5\%), Indonesia (4\%), Uganda (4\%), and other countries with a contribution of $1 \%$ (Figure 4 ). In the selected articles, 10 improvement programs located in different countries were mentioned, containing information with the potential for genetic improvement for the resistance of Musa spp. to Fusarium wilt (Figure 4).

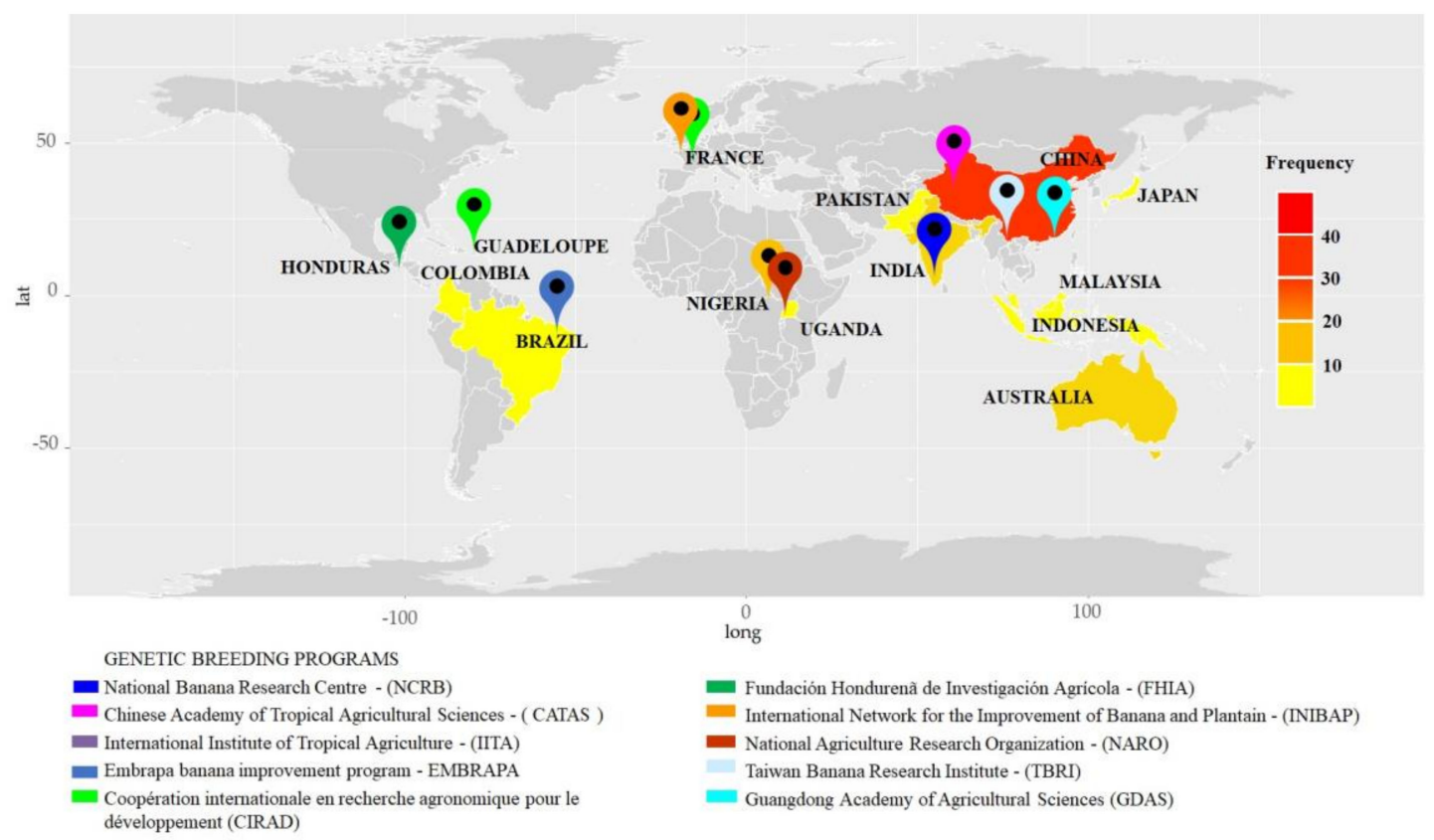

Figure 4. Frequency of articles on genetic improvement of Musa spp. to Fusarium wilt published in the last ten years in different countries and genetic breeding programs of the banana mentioned. The light yellow tones indicate a frequency below $10 \%$ of the articles considered in this review; the intermediate tones indicate frequencies between 10 and $30 \%$, and the intense red tones indicate frequencies above $40 \%$. The location icons indicate the locations of Musa breeding programs identified by the colors. The map was plotted in R, using the packages maps, ggmap, geosphere, Eurostat, GADMTools, country code and ggplot2. lat: latitude; long: longitude. 
Among the improvement programs cited, those that worked with crossbreeding to develop resistant cultivars were as follows: Honduras Foundation for Agricultural Research (Fundación Hondureña de Investigación Agrícola-FHIA), located in Honduras; Centre Africain de Recherches sur Bananiers et Plantains (CARBAP) in Cameroon; the International Institute of Tropical Agriculture (IITA) in Nigeria and Uganda; National Improvement Program of the Brazilian Agricultural Research Corporation (EMBRAPA) in Brazil; National Banana Research Center (NCRB) in India; National Research Organization (NARO) in Uganda; and Centre de Coopération Internationale en Recherche Agronomique pour le Développement (CIRAD) in Guadeloupe, French Antilles (Figure 4). In contrast, the improvement programs Taiwan Banana Research Institute (TBRI) and Guangdong Academy of Agricultural Sciences (GDAAS) in China worked with somaclonal variants and biotechnology.

\subsection{Main Methods and Tools}

Concerning the fungal races, the highest number of articles addressed specific studies with FOC TR $4(57 \%), 25.8 \%$ of the studies dealt only with FOC R1, and $10.1 \%$ of the articles performed comparative studies between FOC TR4 and FOC R1 (Figure 5A). Other studies with lower numbers conducted studies on subtropical race 4 (FOC STR4) (3.2\%), FOC subtropical race 4 (STR4) and Foc TR4 (2.2\%), and FOC R1 and FOC STR4 (1\%) (Figure 5A). The highest frequency of articles was related to in silico $(42.1 \%)$ and in vitro $(32.6 \%)$ studies, followed by studies performed only in the greenhouse $(12.6 \%)$, in the greenhouse and the field $(5.3 \%)$, in the field only $(4.2 \%)$, in the glasshouse $(2.1 \%)$, and in other places $(1 \%)$ (Figure 5B).

a)

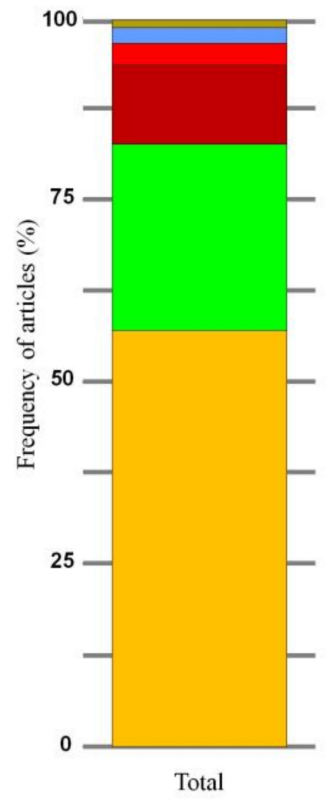

b)

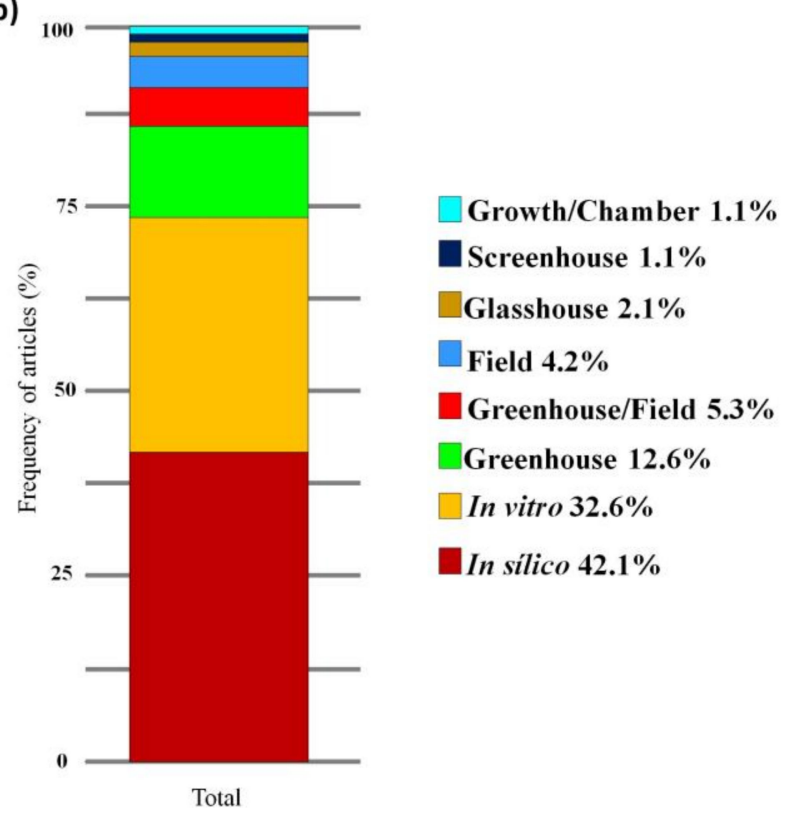

Figure 5. Stacked bar chart of the frequency of articles with different races of Fusarium oxysporum $\mathrm{f}$. sp. cubense in the past ten years (a). Places of achievement of work in articles on the improvement of banana plants to Fusarium wilt carried out in the last 10 years (b). R1: race 1; STR4: subtropical race 4; TR4: tropical race 4.

To evaluate Fusarium wilt symptoms, 26 scales were cited, divided among rhizomediscoloration symptoms, leaf-yellowing symptoms, and pseudostem division (Table 2). We found that $37 \%(n=36)$ of studies adopted a scoring scale for external or internal Fusarium wilt symptoms (Table 2). According to the highest frequency of articles, the most-used scoring grades were from 1 to 6 for rhizome-discoloration and leaf-yellowing symptoms 
and from 1 to 3 for pseudostem division (Table 2). The most frequently cited scales were those of [65-68].

Table 2. Scales of grades for assessing symptoms of Fusarium oxysporum f. sp. cubense reported in articles on banana breeding to Fusarium wilt conducted in the last ten years.

\begin{tabular}{|c|c|c|c|c|}
\hline \multirow{3}{*}{ Article } & \multirow{2}{*}{$\begin{array}{c}\text { Internal Symptoms } \\
\text { Rhizome } \\
\text { Discoloration }\end{array}$} & \multicolumn{2}{|c|}{ External Symptoms } & \multirow{3}{*}{ Scale Reference } \\
\hline & & Yellowing of the Leaf & Pseudostem Division & \\
\hline & \multicolumn{3}{|c|}{ Degrees of Scale } & \\
\hline Yip et al. [69] & $0-3$ & & & {$[69]^{*}$} \\
\hline Orr et al. [70] & $1-6$ & & & [71] \\
\hline Chen et al. [72] & $1-8$ & & & [68] \\
\hline Warman and Aitken [46] & $1-6$ & & & [66] \\
\hline Baharum et al. [73] & $1-8$ & & & [68] \\
\hline Zhang et al. [74] & $0-4$ & $0-4$ & & {$[75,76]$} \\
\hline Zuo et al. [77] & $1-5$ & & & {$[77]^{*}$} \\
\hline Ribeiro et al. [78] & $0-5$ & $0-4$ & & {$[67,79]$} \\
\hline Wei et al. [80] & & $0-4$ & & {$[80]^{*}$} \\
\hline Garcez et al. [81] & $0-5$ & $0-5$ & & {$[67,82]$} \\
\hline Li et al. [75] & $0-3$ & $0-3$ & & {$[75]^{*}$} \\
\hline Ghag et al. [83] & & $1-6$ & & [66] \\
\hline Smith et al. [84] & $1-6$ & & & {$[65,85]$} \\
\hline Mohandas et al. [86] & $1-6$ & $0-5$ & & {$[65,87]$} \\
\hline Ting et al. [88] & & $0-5$ & & {$[88]^{*}$} \\
\hline Paul et al. [89] & & $1-5$ & $1-3$ & [89]* \\
\hline Sun et al. [90] & $1-5$ & $1-5$ & & {$[64,91]$} \\
\hline Wu et al. [92] & & $1-6$ & & {$[92]^{* *}$} \\
\hline Ssali et al. [93] & & $1-6$ & & [94] \\
\hline Li et al. [95] & $0-4$ & $0-5$ & & {$[95]^{*}$} \\
\hline Ghag et al. [96] & & $1-6$ & & {$[96]^{*}$} \\
\hline Saraswathi et al. [97] & $1-5$ & $1-5$ & & {$[66,91]$} \\
\hline Ghag et al. [98] & & $1-6$ & & [83] \\
\hline Sun et al. [76] & & $0-4$ & & {$[76]^{*}$} \\
\hline Wu et al. [99] & & $1-6$ & & {$[99]^{* *}$} \\
\hline Magambo et al. [100] & & $1-5$ & $1-3$ & [68] \\
\hline Smith et al. [101] & $1-6$ & & & [65] \\
\hline García-Bastidas et al. [102] & $1-6$ & $1-4$ & & {$[102]^{*}$} \\
\hline Arinaitwe et al. [31] & $1-5$ & $1-6$ & $1-3$ & [71] \\
\hline Cheng et al. [103] & $1-8$ & & & [68] \\
\hline Gonçalves et al. [33] & $1-5$ & $1-6$ & & {$[67,104]$} \\
\hline Buregyeya et al. [105] & $1-6$ & & $1-3$ & [94] \\
\hline Sunisha et al. [106] & & $1-5$ & $1-3$ & [89] \\
\hline Rocha et al. [107] & $1-5$ & $1-4$ & & [104] \\
\hline Ahmad et al. [108] & $1-6$ & $1-4$ & & [102] \\
\hline
\end{tabular}


Among the main methods used for obtainment or characterization of plants resistant to Fusarium wilt, gene expression analysis represented $33 \%$ of the selected articles, followed by transgenesis $(16 \%)$, symptomatology $(13 \%)$, and resistance induction $(11 \%)$ (Figure 6). In related articles, the other methods were classified as molecular markers (5\%), symptomatology associated with the agronomic characterization of banana genotypes (5\%), in vitro mutagenesis $(4 \%)$, enzyme activity $(3 \%)$, protein analysis and expression $(3 \%)$, hybridization by crossbreeding ( $2 \%)$, and methods of somaclonal variation, clone selection, and somatic embryogenesis, each with a $1 \%$ frequency (Figure 6).

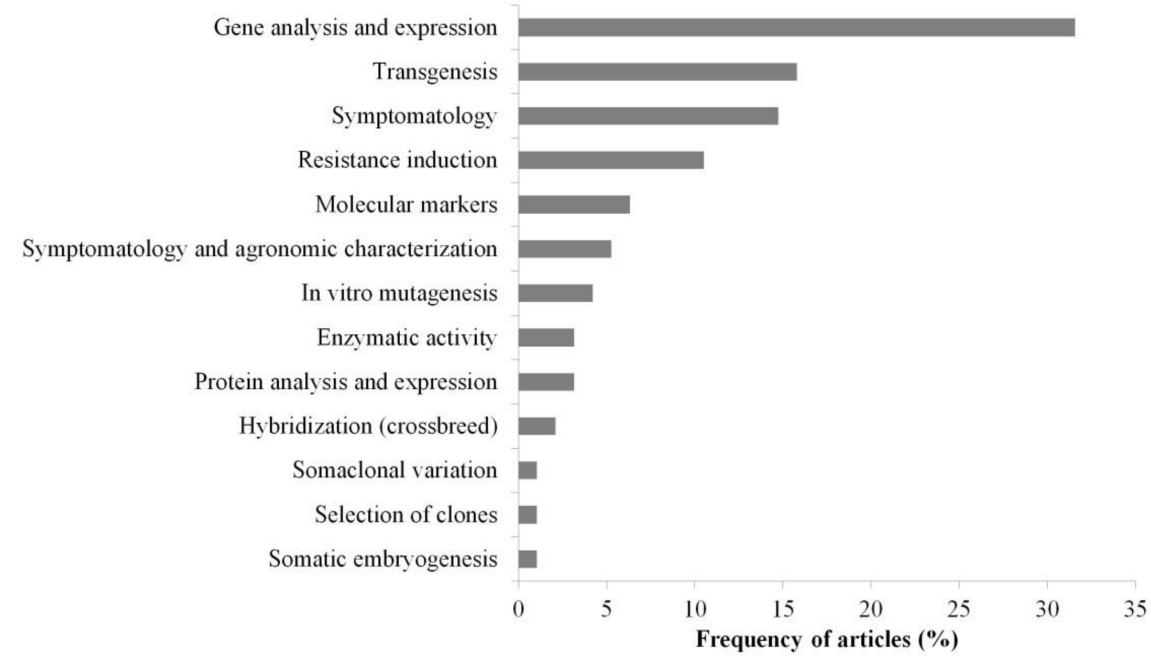

Figure 6. Banana plant breeding techniques used to supplant Fusarium wilt in articles published in the last 10 years.

Among the tools used for the analysis and characterization of plants resistant to Fusarium wilt, the frequency of articles that employed analysis of reverse transcriptionPCR (RT-qPCR) and PCR was the highest (35\%). Analyses using bioinformatics tools were in $23 \%$ of the articles, and tissue culture represented 13\% (Figure 7). Other tools adopted included the genetic transformation of the fungus with the GFP gene, the infection process by FOC strains $(7 \%)$, banana transcriptome $(7 \%)$, and phylogenetic analysis $(7 \%)$. In addition to these tools, there was also a portion of articles using histochemistry and/or histology (6\%) and other tools with a lower frequency (Figure 7).

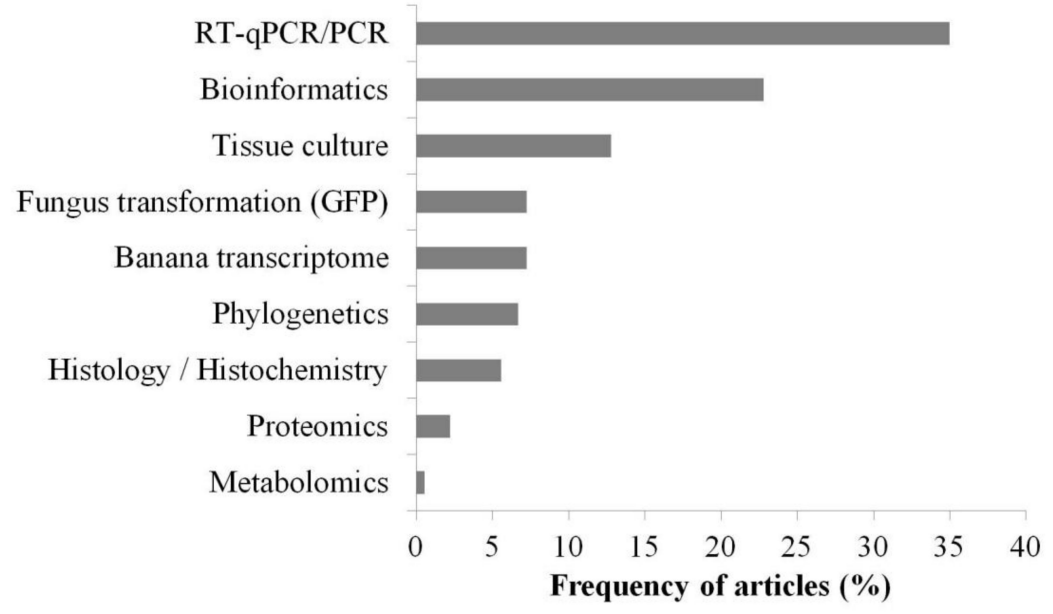

Figure 7. Frequency of articles associated with the main tools used in studies on banana plant breeding to Fusarium wilt in the last 10 years. The frequency considered that more than one tool was used per article. RT-qPCR/PCR: reverse transcription-PCR/polymerase chain reaction/ GFP: green fluorescent protein. 
Some articles used molecular markers associated with wilting resistance: Silva et al. [109], Wang et al. [110], and Wang et al. [51]. Among the markers associated with the resistance to FOC TR4, seven were from sequence characterized amplified region (SCAR)-type. One marker was associated with the susceptibility to FOC R1 [111] (Table 3). One random amplified polymorphic DNA (RAPD) molecular marker associated with the resistance to FOC R1 was found by Ghag et al. [98] (Table 3).

Table 3. Molecular markers associated with banana breeding strategies to Fusarium wilt in articles carried out in the last ten years.

\begin{tabular}{cccc}
\hline Name & Type & Function & Citation \\
\hline ScaU1001 & SCAR & Resistance to FOC TR4 & {$[109]$} \\
\hline SuscPD & SCAR & Susceptibility to FOC 1 & {$[111]$} \\
\hline Lipoxygenase (gene) & RAPD & Resistance to FOC 1 & {$[98]$} \\
\hline $\begin{array}{l}\text { ScaU1001 } \\
\text { ScaS0901 }\end{array}$ & SCAR & Resistance to FOC TR4 & {$[110]$} \\
\hline SC1/SC2 & & & \\
SC3/SC4 & & & \\
SC5/SC6 & Resistance to FOC TR4 & \\
SC7/SC8 & SCAR & & \\
\hline SCAR: sequence characterized amplified region; RAPD: random amplified polymorphic DNA. &
\end{tabular}

\subsection{Resistance Sources}

In the set of selected articles, many sources of resistance to Fusarium wilt were found for different FOC races (Table 4). Of the sources reported as resistant, 38\% were triploid (AAA genome), 33\% were diploid (AA genome), 12\% were triploid (AAB genome), and 8\% were tetraploid (AAAB genome); other genomes reported had a frequency of less than $5 \%$ (Figure 8 and Table 4 ).

Table 4. Sources of resistance to Fusarium oxysporum f. sp. cubense characterized in articles on the improvement of banana to Fusarium wilt carried out in the last ten years.

\begin{tabular}{|c|c|c|c|c|c|c|}
\hline $\begin{array}{c}\text { Musa } \\
\text { Germplasm }\end{array}$ & $\begin{array}{l}\text { Musa } \\
\text { Genome }\end{array}$ & Race & $\begin{array}{l}\text { Level of Tolerance or } \\
\text { Resistance to Races }\end{array}$ & $\begin{array}{l}\text { Institution and Location/Country } \\
\text { Where Germplasm Was Screened }\end{array}$ & $\begin{array}{l}\text { Known Use to Mitigate } \\
\text { Fusarium Impact }\end{array}$ & References \\
\hline M53 & AA & Race 1 & $\mathrm{R}$ & $\begin{array}{l}\text { Embrapa cassava and fruit } \\
\text { growing, Brazil }\end{array}$ & In breeding programs & {$[33,78,107]$} \\
\hline Birmanie & AA & Race 1 & $\mathrm{R}$ & $\begin{array}{l}\text { Embrapa cassava and fruit } \\
\text { growing, Brazil }\end{array}$ & In breeding programs & {$[78,107]$} \\
\hline PA Songkla & AA & Race 1 & $\mathrm{R}$ & $\begin{array}{l}\text { Embrapa cassava and fruit } \\
\text { growing, Brazil }\end{array}$ & In breeding programs & [78] \\
\hline Pirua & AAA & Race 1 & $\mathrm{R}$ & $\begin{array}{l}\text { Embrapa cassava and fruit } \\
\text { growing, Brazil }\end{array}$ & Brazil & [78] \\
\hline Imperial & AAA & Race 1 & $\mathrm{R}$ & $\begin{array}{l}\text { Embrapa cassava and fruit } \\
\text { growing, Brazil }\end{array}$ & Brazil & [78] \\
\hline Poyo & AAA & Race 1 & $\mathrm{R}$ & $\begin{array}{l}\text { Embrapa cassava and fruit } \\
\text { growing, Brazil [78], DAFF, } \\
\text { Australia [84] }\end{array}$ & Brazil, Africa & {$[78,84]$} \\
\hline BRS Vitória & $\mathrm{AAAB}$ & Race 1 & $\mathrm{R}$ & $\begin{array}{l}\text { Embrapa cassava and fruit } \\
\text { growing, Brazil }\end{array}$ & Brazil & [107] \\
\hline Ambei & AA & Race 1 & $\mathrm{R}$ & $\begin{array}{l}\text { Embrapa cassava and fruit } \\
\text { growing, Brazil }\end{array}$ & In breeding programs & [78] \\
\hline Walebo & AAA & Race 1 & $\mathrm{R}$ & $\begin{array}{l}\text { Embrapa cassava and fruit } \\
\text { growing, Brazil }\end{array}$ & Brazil & [78] \\
\hline
\end{tabular}


Table 4. Cont.

\begin{tabular}{|c|c|c|c|c|c|c|}
\hline $\begin{array}{c}\text { Musa } \\
\text { Germplasm }\end{array}$ & $\begin{array}{l}\text { Musa } \\
\text { Genome }\end{array}$ & Race & $\begin{array}{l}\text { Level of Tolerance or } \\
\text { Resistance to Races }\end{array}$ & $\begin{array}{l}\text { Institution and Location/Country } \\
\text { Where Germplasm Was Screened }\end{array}$ & $\begin{array}{l}\text { Known Use to Mitigate } \\
\text { Fusarium Impact }\end{array}$ & References \\
\hline $\begin{array}{l}\text { Kongo FRF } \\
1286\end{array}$ & AAA & Race 1 & $\mathrm{R}$ & $\begin{array}{l}\text { Embrapa cassava and fruit } \\
\text { growing, Brazil }\end{array}$ & Brazil & [78] \\
\hline $\begin{array}{l}\text { Pisang } \\
\text { Nangka }\end{array}$ & $\mathrm{AAB}$ & Race 1 & $\mathrm{R}$ & $\begin{array}{l}\text { Embrapa cassava and fruit } \\
\text { growing, Brazil }\end{array}$ & Brazil, Africa, Australia & [78] \\
\hline Pisang Jaran & AA & Race 1 & $\mathrm{R}$ & $\begin{array}{c}\text { Embrapa cassava and fruit } \\
\text { growing, Brazil }\end{array}$ & In breeding programs & [78] \\
\hline Tjau Lagada & AA & Race 1 & $\mathrm{R}$ & $\begin{array}{l}\text { Embrapa cassava and fruit } \\
\text { growing, Brazil }[33,78]\end{array}$ & In breeding programs & {$[33,78]$} \\
\hline Mangana & AA & Race 1 & $\mathrm{R}$ & $\begin{array}{l}\text { Embrapa cassava and fruit } \\
\text { growing, Brazil }\end{array}$ & In breeding programs & [78] \\
\hline Pisang Pipit & AAA & Race 1 & $\mathrm{R}$ & $\begin{array}{l}\text { Embrapa cassava and fruit } \\
\text { growing, Brazil }\end{array}$ & In breeding programs & {$[78]$} \\
\hline $\begin{array}{l}\text { Pisang Rojo } \\
\text { Uter }\end{array}$ & AA & Race 1 & $\mathrm{R}$ & $\begin{array}{l}\text { Embrapa cassava and fruit } \\
\text { growing, Brazil }\end{array}$ & In breeding programs & [78] \\
\hline 2803-01 & AA & Race 1 & $\mathrm{R}$ & $\begin{array}{l}\text { Embrapa cassava and fruit } \\
\text { growing, Brazil }\end{array}$ & In breeding programs & [78] \\
\hline $\begin{array}{l}\text { GN. P. } \\
\text { Formoso }\end{array}$ & AAA & Race 1 & $\mathrm{R}$ & $\begin{array}{l}\text { Embrapa cassava and fruit } \\
\text { growing, Brazil }\end{array}$ & In breeding programs & [109] \\
\hline $\begin{array}{l}\text { Pisang } \\
\text { Tongat }\end{array}$ & AA & Race 1 & $\mathrm{R}$ & $\begin{array}{l}\text { Embrapa cassava and fruit } \\
\text { growing, Brazil }\end{array}$ & In breeding programs & [78] \\
\hline $\begin{array}{l}\text { Mchare } \\
\text { cultivars }\end{array}$ & AA & Race 1 & $\mathrm{R}$ & $\begin{array}{l}\text { Stellenbosch University, South } \\
\text { Africa (Arusha, Tanzania) }\end{array}$ & Africa & [112] \\
\hline $\begin{array}{l}\text { Mchare } \\
\text { hybrids }\end{array}$ & AA & Race 1 & $\mathrm{R}$ & $\begin{array}{l}\text { Stellenbosch University, South } \\
\text { Africa (Arusha, Tanzania) }\end{array}$ & Africa & [112] \\
\hline $\begin{array}{l}\text { NARITA } \\
\text { hybrids }\end{array}$ & AA & Race 1 & $\mathrm{R}$ & $\begin{array}{l}\text { Stellenbosch University, South } \\
\text { Africa (Kawanda, Uganda) }\end{array}$ & Africa & [112] \\
\hline Figo Cinza & $\mathrm{ABB}$ & Race 1 & $\mathrm{R}$ & $\begin{array}{l}\text { Embrapa cassava and fruit } \\
\text { growing, Brazil [78] } \\
\text { Banana Germplasm Bank of the } \\
\text { Itajaí Research Station [111] }\end{array}$ & Brazil & {$[78,111]$} \\
\hline M-61 & AAA & Race 1 & $\mathrm{R}$ & $\begin{array}{l}\text { Embrapa cassava and fruit } \\
\text { growing, Brazil }\end{array}$ & In breeding programs & [78] \\
\hline $\begin{array}{l}\text { Nanicão } \\
\text { Magario }\end{array}$ & AAA & Race 1 & $\mathrm{R}$ & $\begin{array}{l}\text { Embrapa cassava and fruit } \\
\text { growing, Brazil }\end{array}$ & Brazil & [78] \\
\hline Buitenzorg & AA & Race 1 & $\mathrm{R}$ & $\begin{array}{l}\text { Embrapa cassava and fruit } \\
\text { growing, Brazil }\end{array}$ & In breeding programs & [78] \\
\hline BRS Platina & $\mathrm{AAAB}$ & Race 1 & $\mathrm{R}$ & $\begin{array}{c}\text { Embrapa cassava and fruit } \\
\text { growing, Brazil [33,78,107] } \\
\text { Itajaí Research Station, Brazil [111] }\end{array}$ & Brazil & $\begin{array}{l}{[33,78,107} \\
111]\end{array}$ \\
\hline Nanica & AAA & Race 1 & $\mathrm{R}$ & $\begin{array}{l}\text { Embrapa cassava and fruit } \\
\text { growing, Brazil }\end{array}$ & Brazil & $\begin{array}{c}{[78,107,} \\
109]\end{array}$ \\
\hline $\begin{array}{l}\text { Pisang } \\
\text { Ustrali }\end{array}$ & $\mathrm{AAB}$ & Race 1 & $\mathrm{R}$ & $\begin{array}{c}\text { Embrapa cassava and fruit } \\
\text { growing, Brazil }\end{array}$ & In breeding programs & [78] \\
\hline Markatooa & AAA & Race 1 & $\mathrm{R}$ & $\begin{array}{l}\text { Embrapa cassava and fruit } \\
\text { growing, Brazil }\end{array}$ & In breeding programs & [78] \\
\hline Robusta & AAA & Race 1 & $\mathrm{R}$ & $\begin{array}{l}\text { Embrapa cassava and fruit } \\
\text { growing, Brazil }\end{array}$ & In breeding programs & [78] \\
\hline $\begin{array}{l}\text { BRS Pacovan } \\
\text { Ken }\end{array}$ & AAAB & Race 1 & $\mathrm{R}$ & $\begin{array}{l}\text { Embrapa cassava and fruit } \\
\text { growing, Brazil }\end{array}$ & Brazil & {$[78,107]$} \\
\hline $\begin{array}{c}\text { BRS } \\
\text { Princesa }\end{array}$ & AAAB & Race 1 & $\mathrm{R}$ & $\begin{array}{l}\text { Federal Institute of the Triangulo } \\
\text { Mineiro, Brazil [74], Embrapa cassava } \\
\text { and fruit growing, Brazil [33,107] }\end{array}$ & Brazil & {$[33,81,107]$} \\
\hline
\end{tabular}


Table 4. Cont.

\begin{tabular}{|c|c|c|c|c|c|c|}
\hline $\begin{array}{c}\text { Musa } \\
\text { Germplasm }\end{array}$ & $\begin{array}{l}\text { Musa } \\
\text { Genome }\end{array}$ & Race & $\begin{array}{l}\text { Level of Tolerance or } \\
\text { Resistance to Races }\end{array}$ & $\begin{array}{l}\text { Institution and Location/Country } \\
\text { Where Germplasm Was Screened }\end{array}$ & $\begin{array}{l}\text { Known Use to Mitigate } \\
\text { Fusarium Impact }\end{array}$ & References \\
\hline BRS Japira & $\mathrm{AAAB}$ & Race 1 & $\mathrm{R}$ & $\begin{array}{l}\text { Federal Institute of the Triangulo } \\
\text { Mineiro, Brazil [81] Embrapa cassava } \\
\text { and fruit growing, Brazil [107] }\end{array}$ & Brazil & {$[81,107]$} \\
\hline BRS Tropical & $\mathrm{AAAB}$ & Race 1 & $\mathrm{R}$ & $\begin{array}{l}\text { Federal Institute of the Triangulo } \\
\text { Mineiro, Brazil }\end{array}$ & Brazil & [81] \\
\hline $\begin{array}{l}\text { Grand } \\
\text { Naine }\end{array}$ & AAA & Race 1 & $\mathrm{R}$ & $\begin{array}{l}\text { Embrapa cassava and fruit } \\
\text { growing, Brazil }[33,78,107,109], \\
\text { Federal University of Santa } \\
\text { Catarina, Brazil [111] }\end{array}$ & Cavendish for export & $\begin{array}{c}{[33,78,107,} \\
109,111]\end{array}$ \\
\hline Nanicão & AAA & Race 1 & $\mathrm{R}$ & $\begin{array}{c}\text { Embrapa cassava and fruit growing, } \\
\text { Brazil [78,111], Federal University of } \\
\text { Santa Catarina, Brazil [111] }\end{array}$ & Brazil & $\begin{array}{c}{[78,109,} \\
111]\end{array}$ \\
\hline $\begin{array}{l}\text { SCS452 } \\
\text { Corupá }\end{array}$ & AAA & Race 1 & $\mathrm{R}$ & $\begin{array}{l}\text { Federal University of Santa } \\
\text { Catarina, Brazil }\end{array}$ & Brazil & [111] \\
\hline Zellig & AAA & Race 1 & $\mathrm{R}$ & $\begin{array}{l}\text { Federal University of Santa } \\
\text { Catarina, Brazil }\end{array}$ & Brazil & [111] \\
\hline Figo & $\mathrm{ABB}$ & Race 1 & $\mathrm{R}$ & $\begin{array}{c}\text { Embrapa cassava and fruit growing, } \\
\text { Brazil [78], Federal University of } \\
\text { Santa Catarina, Brazil [111] }\end{array}$ & In breeding programs & {$[78,111]$} \\
\hline FHIA-17 & AAAA & Race 1 & $\mathrm{R}$ & DAFF, Australia & Honduras, Brazil & [84] \\
\hline SH-3640.10 & $\mathrm{AAAB}$ & Race 1 & $\mathrm{R}$ & DAFF, Australia & $\begin{array}{c}\text { Honduras, Brazil, } \\
\text { Mozambique, Cameroon }\end{array}$ & [84] \\
\hline Long Tavoy & * & Race 1 & $\mathrm{R}$ & $\begin{array}{l}\text { University of Malaya, Kuala } \\
\text { Lumpur, Malaysia }\end{array}$ & In breeding programs & [31] \\
\hline Kasaska & * & Race 1 & $\mathrm{R}$ & $\begin{array}{l}\text { University of Malaya, Kuala } \\
\text { Lumpur, Malaysia }\end{array}$ & In breeding programs & [31] \\
\hline Monyet & * & Race 1 & $\mathrm{R}$ & $\begin{array}{l}\text { University of Malaya, Kuala } \\
\text { Lumpur, Malaysia }\end{array}$ & In breeding programs & {$[31]$} \\
\hline $\begin{array}{l}\text { Mwitu } \\
\text { Pemba }\end{array}$ & * & Race 1 & $\mathrm{R}$ & $\begin{array}{l}\text { University of Malaya, Kuala } \\
\text { Lumpur, Malaysia }\end{array}$ & In breeding programs & [31] \\
\hline $\begin{array}{l}\text { Hom Thong } \\
\text { Mokho }\end{array}$ & AAA & Race 1 & $\mathrm{R}$ & $\begin{array}{c}\text { Department of Agriculture and } \\
\text { Fisheries (DAF), Queensland, } \\
\text { Australia }\end{array}$ & Australia & [101] \\
\hline $\begin{array}{l}\text { Mambee } \\
\text { Thu }\end{array}$ & AA & Race 1 & $\mathrm{R}$ & $\begin{array}{l}\text { Embrapa cassava and fruit } \\
\text { growing, Brazil }\end{array}$ & In breeding programs & [78] \\
\hline PV03-79 & AAAB & Race 1 & $\mathrm{R}$ & $\begin{array}{l}\text { Embrapa cassava and fruit } \\
\text { growing, Brazil }\end{array}$ & In breeding programs & [78] \\
\hline $\begin{array}{c}\text { Terra } \\
\text { Maranhão }\end{array}$ & $\mathrm{AAB}$ & Race 1 & $\mathrm{R}$ & $\begin{array}{l}\text { Embrapa cassava and fruit } \\
\text { growing, Brazil }\end{array}$ & Brazil & [107] \\
\hline Williams & AAA & Race 1 & $\mathrm{R}$ & $\begin{array}{c}\text { DAFF, Australia [101], Federal } \\
\text { University of Santa Catarina, } \\
\text { Brazil [111] }\end{array}$ & Cavendish for export & {$[101,111]$} \\
\hline Williams & AAA & STR4 & SS & $\begin{array}{l}\text { University of Queensland, } \\
\text { Australia }\end{array}$ & Cavendish for export & [72] \\
\hline SH-3217 & AA & STR4 & $\mathrm{R}$ & $\begin{array}{l}\text { University of Queensland, } \\
\text { Australia }\end{array}$ & In breeding programs & [72] \\
\hline Ma250 & AA & STR4 & $\mathrm{R}$ & $\begin{array}{c}\text { University of Queensland, } \\
\text { Australia }\end{array}$ & In breeding programs & [72] \\
\hline $\begin{array}{c}\text { Pisang } \\
\text { Bangkahulu }\end{array}$ & AA & STR4 & $\mathrm{R}$ & $\begin{array}{l}\text { University of Queensland, } \\
\text { Australia }\end{array}$ & In breeding programs & [72] \\
\hline $\begin{array}{c}\text { M61 } \\
\text { Guadelope }\end{array}$ & * & STR4 & SS & $\begin{array}{l}\text { University of Queensland, } \\
\text { Australia }\end{array}$ & In breeding programs & [72] \\
\hline
\end{tabular}


Table 4. Cont.

\begin{tabular}{|c|c|c|c|c|c|c|}
\hline $\begin{array}{l}\text { Musa } \\
\text { Germplasm }\end{array}$ & $\begin{array}{l}\text { Musa } \\
\text { Genome }\end{array}$ & Race & $\begin{array}{l}\text { Level of Tolerance or } \\
\text { Resistance to Races }\end{array}$ & $\begin{array}{l}\text { Institution and Location/Country } \\
\text { Where Germplasm Was Screened }\end{array}$ & $\begin{array}{l}\text { Known Use to Mitigate } \\
\text { Fusarium Impact }\end{array}$ & References \\
\hline CAM-020 & AAA & STR4 & S & $\begin{array}{c}\text { University of Queensland, } \\
\text { Australia }\end{array}$ & In breeding programs & [72] \\
\hline SH-3142 & AA & TR4 & SS & $\begin{array}{l}\text { (IFTR-GDAAS), } \\
\text { Guangzhou, China }\end{array}$ & In breeding programs & [77] \\
\hline $\begin{array}{l}\text { FHIA-1 } \\
\text { ("Gold } \\
\text { Finger") }\end{array}$ & $\mathrm{AAAB}$ & TR4 & S & GDAAS, Guangzhou, China & $\begin{array}{c}\text { Australia, Brazil, } \\
\text { Mexico, Colombia, EUA }\end{array}$ & [75] \\
\hline GCTCV-119 & AAA & TR4 & HR & $\begin{array}{c}\text { Guangdong Academy of } \\
\text { Agricultural Sciences, } \\
\text { Guangzhou, China }\end{array}$ & $\begin{array}{c}\text { China, Taiwan, The } \\
\text { Philippines, } \\
\text { Mozambique. }\end{array}$ & [92] \\
\hline $\begin{array}{c}\text { M61 } \\
\text { Guadeloupe }\end{array}$ & * & TR4 & $\mathrm{R}$ & $\begin{array}{l}\text { University of Queensland, } \\
\text { Australia }\end{array}$ & In breeding programs & {$[72]$} \\
\hline CAM-020 & AAA & TR4 & $\mathrm{R}$ & $\begin{array}{l}\text { University of Queensland, } \\
\text { Australia }\end{array}$ & In breeding programs & [72] \\
\hline Ibwi E & AAA & TR4 & $\mathrm{R}$ & $\begin{array}{l}\text { (IFTR-GDAAS), } \\
\text { Guangzhou, China }\end{array}$ & EAHBs & [77] \\
\hline $\begin{array}{l}\text { Igitsiri } \\
\text { (Intuntu) }\end{array}$ & AAA & TR4 & $\mathrm{R}$ & $\begin{array}{l}\text { (IFTR-GDAAS), } \\
\text { Guangzhou, China }\end{array}$ & EAHBs & [77] \\
\hline Ingagara & AAA & TR4 & $\mathrm{R}$ & $\begin{array}{l}\text { (IFTR-GDAAS), } \\
\text { Guangzhou, China }\end{array}$ & EAHBs & [77] \\
\hline Inkira & AAA & TR4 & $\mathrm{R}$ & $\begin{array}{l}\text { (IFTR-GDAAS), } \\
\text { Guangzhou, China }\end{array}$ & EAHBs & [77] \\
\hline Intokatoke & AAA & TR4 & $\mathrm{R}$ & $\begin{array}{l}\text { (IFTR-GDAAS), } \\
\text { Guangzhou, China }\end{array}$ & EAHBs & [77] \\
\hline Kazirakwe & AAA & TR4 & $\mathrm{R}$ & $\begin{array}{l}\text { (IFTR-GDAAS), } \\
\text { Guangzhou, China }\end{array}$ & EAHBs & [77] \\
\hline Mbwazirume & AAA & TR4 & HR & $\begin{array}{l}\text { (IFTR-GDAAS), } \\
\text { Guangzhou, China }\end{array}$ & Africa & [77] \\
\hline Akpakpak & $\mathrm{AAB}$ & TR4 & HR & $\begin{array}{l}\text { (IFTR-GDAAS), } \\
\text { Guangzhou, China }\end{array}$ & Africa & [77] \\
\hline $\begin{array}{l}\text { Curaré } \\
\text { Enano }\end{array}$ & $\mathrm{AAB}$ & TR4 & $\mathrm{R}$ & $\begin{array}{l}\text { (IFTR-GDAAS), } \\
\text { Guangzhou, China }\end{array}$ & Africa & [77] \\
\hline $\begin{array}{l}\text { Obino } \\
\text { l'Ewai }\end{array}$ & $\mathrm{AAB}$ & TR4 & $\mathrm{R}$ & $\begin{array}{l}\text { (IFTR-GDAAS), } \\
\text { Guangzhou, China }\end{array}$ & Africa & [77] \\
\hline $\begin{array}{l}\text { Obubit } \\
\text { Ntanga }\end{array}$ & $\mathrm{AAB}$ & TR4 & $\mathrm{R}$ & $\begin{array}{l}\text { (IFTR-GDAAS), } \\
\text { Guangzhou, China }\end{array}$ & Africa & [77] \\
\hline $\begin{array}{l}\text { Orishele } \\
\text { False Horn }\end{array}$ & $\mathrm{AAB}$ & TR4 & HR & $\begin{array}{l}\text { (IFTR-GDAAS), } \\
\text { Guangzhou, China }\end{array}$ & Africa & {$[77]$} \\
\hline $\begin{array}{l}\text { Pisang } \\
\text { Ceylan }\end{array}$ & $\mathrm{AAB}$ & TR4 & $\mathrm{R}$ & $\begin{array}{l}\text { (IFTR-GDAAS), } \\
\text { Guangzhou, China }\end{array}$ & Africa & [77] \\
\hline Pisang Rajah & $\mathrm{AAB}$ & TR4 & $\mathrm{R}$ & $\begin{array}{l}\text { (IFTR-GDAAS), } \\
\text { Guangzhou, China }\end{array}$ & Africa & [77] \\
\hline $\begin{array}{c}\text { Musa } \\
\text { itinerans }\end{array}$ & * & TR4 & $\mathrm{HR}$ & $\begin{array}{l}\text { (IFTR-GDAAS), } \\
\text { Guangzhou, China }\end{array}$ & In breeding programs & [77] \\
\hline $\begin{array}{l}\text { CIRAD930/DH } \\
\text { Pahang }\end{array}$ & AA & TR4 & HR & $\begin{array}{l}\text { (IFTR-GDAAS), } \\
\text { Guangzhou, China }\end{array}$ & In breeding programs & [77] \\
\hline NBA 14 & AA & TR4 & $\mathrm{R}$ & $\begin{array}{l}\text { (IFTR-GDAAS), } \\
\text { Guangzhou, China }\end{array}$ & In breeding programs & [77] \\
\hline Banksii & AA & TR4 & $\mathrm{R}$ & $\begin{array}{l}\text { (IFTR-GDAAS), } \\
\text { Guangzhou, China }\end{array}$ & In breeding programs & [77] \\
\hline Maia Oa & AA & TR4 & $\mathrm{R}$ & $\begin{array}{l}\text { (IFTR-GDAAS), } \\
\text { Guangzhou, China }\end{array}$ & In breeding programs & [77] \\
\hline
\end{tabular}


Table 4. Cont.

\begin{tabular}{|c|c|c|c|c|c|c|}
\hline $\begin{array}{c}\text { Musa } \\
\text { Germplasm }\end{array}$ & $\begin{array}{l}\text { Musa } \\
\text { Genome }\end{array}$ & Race & $\begin{array}{l}\text { Level of Tolerance or } \\
\text { Resistance to Races }\end{array}$ & $\begin{array}{l}\text { Institution and Location/Country } \\
\text { Where Germplasm Was Screened }\end{array}$ & $\begin{array}{l}\text { Known Use to Mitigate } \\
\text { Fusarium Impact }\end{array}$ & References \\
\hline Zebrina & AA & TR4 & SS & $\begin{array}{l}\text { (IFTR-GDAAS), } \\
\text { Guangzhou, China }\end{array}$ & In breeding programs & [77] \\
\hline Pa (Rayong) & AA & TR4 & $\mathrm{R}$ & $\begin{array}{l}\text { (IFTR-GDAAS), } \\
\text { Guangzhou, China }\end{array}$ & In breeding programs & [77] \\
\hline Figue Rose & AA & TR4 & $\mathrm{R}$ & $\begin{array}{l}\text { (IFTR-GDAAS), } \\
\text { Guangzhou, China }\end{array}$ & In breeding programs & [77] \\
\hline $\begin{array}{l}\text { Khai (Kamp- } \\
\text { engpeth) }\end{array}$ & AA & TR4 & $\mathrm{R}$ & $\begin{array}{l}\text { (IFTR-GDAAS), } \\
\text { Guangzhou, China }\end{array}$ & In breeding programs & [77] \\
\hline Tani & $\mathrm{BB}$ & TR4 & $\mathrm{R}$ & $\begin{array}{l}\text { (IFTR-GDAAS), } \\
\text { Guangzhou, China }\end{array}$ & In breeding programs & [77] \\
\hline $\begin{array}{l}\text { Pisang } \\
\text { Klutuk } \\
\text { Wulung }\end{array}$ & $\mathrm{BB}$ & TR4 & $\mathrm{R}$ & $\begin{array}{c}\text { (IFTR-GDAAS), } \\
\text { Guangzhou, China }\end{array}$ & In breeding programs & [77] \\
\hline $\begin{array}{l}\text { Musa beccarii } \\
\text { Callimusa }\end{array}$ & * & TR4 & $\mathrm{R}$ & $\begin{array}{l}\text { (IFTR-GDAAS), } \\
\text { Guangzhou, China }\end{array}$ & In breeding programs & [77] \\
\hline $\begin{array}{l}\text { Musa laterita } \\
\text { Rhodochlamys }\end{array}$ & * & TR4 & $\mathrm{R}$ & $\begin{array}{l}\text { (IFTR-GDAAS), } \\
\text { Guangzhou, China }\end{array}$ & In breeding programs, & [77] \\
\hline $\begin{array}{c}\text { Musa } \\
\text { maclayi ssp. }\end{array}$ & * & TR4 & $\mathrm{R}$ & $\begin{array}{l}\text { (IFTR-GDAAS), } \\
\text { Guangzhou, China }\end{array}$ & In breeding programs, & [77] \\
\hline $\begin{array}{l}\text { Khai Thong } \\
\text { Ruang }\end{array}$ & AAA & TR4 & $\mathrm{R}$ & $\begin{array}{l}\text { (IFTR-GDAAS), } \\
\text { Guangzhou, China }\end{array}$ & In breeding programs & [77] \\
\hline Kamaramasenge & $\mathrm{AB}$ & TR4 & $\mathrm{R}$ & $\begin{array}{l}\text { (IFTR-GDAAS), } \\
\text { Guangzhou, China }\end{array}$ & In breeding programs & [77] \\
\hline Rukumamb & $\mathrm{AAB}$ & TR4 & $\mathrm{R}$ & $\begin{array}{l}\text { (IFTR-GDAAS), } \\
\text { Guangzhou, China }\end{array}$ & $\begin{array}{c}\text { Australia, Papua New } \\
\text { Guinea }\end{array}$ & [77] \\
\hline Thap Maeo & $\mathrm{AAB}$ & TR4 & $\mathrm{R}$ & $\begin{array}{l}\text { (IFTR-GDAAS), } \\
\text { Guangzhou, China }\end{array}$ & Brazil, Honduras & [77] \\
\hline Foconah & $\mathrm{AAB}$ & TR4 & $\mathrm{R}$ & $\begin{array}{l}\text { (IFTR-GDAAS), } \\
\text { Guangzhou, China }\end{array}$ & In breeding programs & [77] \\
\hline Poingo & $\mathrm{AAB}$ & TR4 & $\mathrm{R}$ & $\begin{array}{c}\text { (IFTR-GDAAS), } \\
\text { Guangzhou, China }\end{array}$ & In breeding programs & [77] \\
\hline FHIA-21 & $\mathrm{AAAB}$ & TR4 & $\mathrm{R}$ & $\begin{array}{c}\text { (IFTR-GDAAS), } \\
\text { Guangzhou, China [77], DAFF, } \\
\text { Australia [84] }\end{array}$ & In breeding programs & {$[77,84]$} \\
\hline Blue Java & $\mathrm{ABB}$ & TR4 & $\mathrm{R}$ & $\begin{array}{c}\text { (IFTR-GDAAS), } \\
\text { Guangzhou, China [77], Embrapa } \\
\text { cassava and fruit growing, Brazil } \\
\text { [107] }\end{array}$ & China, Africa, Brazil & {$[77,107]$} \\
\hline $\begin{array}{l}\text { Namwa } \\
\text { Khom }\end{array}$ & $\mathrm{ABB}$ & TR4 & HR & $\begin{array}{l}\text { (IFTR-GDAAS), } \\
\text { Guangzhou, China [77], DAF, } \\
\text { Australia [101] }\end{array}$ & China, Africa, Thailand & {$[77,101]$} \\
\hline FHIA-02 & AAAA & TR4 & $\mathrm{R}$ & DAFF, Australia & $\begin{array}{l}\text { Africa, Brazil, Colombia, } \\
\text { Honduras }\end{array}$ & {$[72,84]$} \\
\hline $\begin{array}{l}\text { SH-3362 } \\
\text { ("Pita-16") }\end{array}$ & * & TR4 & $\mathrm{R}$ & DAFF, Australia & In breeding programs & {$[72]$} \\
\hline $\begin{array}{c}\text { M. } \\
\text { yunnanensis }\end{array}$ & * & TR4 & $\mathrm{R}$ & $\begin{array}{c}\text { South China Agricultural } \\
\text { University }\end{array}$ & Wild germplasm & [75] \\
\hline M. basjoo & * & TR4 & $\mathrm{R}$ & $\begin{array}{l}\text { South China Agricultural } \\
\text { University }\end{array}$ & Wild germplasm & [75] \\
\hline $\begin{array}{c}\text { M. } \\
\text { nagensium }\end{array}$ & * & TR4 & $\mathrm{R}$ & $\begin{array}{l}\text { South China Agricultural } \\
\text { University }\end{array}$ & Wild germplasm & [75] \\
\hline
\end{tabular}


Table 4. Cont.

\begin{tabular}{|c|c|c|c|c|c|c|}
\hline $\begin{array}{c}\text { Musa } \\
\text { Germplasm }\end{array}$ & $\begin{array}{c}\text { Musa } \\
\text { Genome }\end{array}$ & Race & $\begin{array}{l}\text { Level of Tolerance or } \\
\text { Resistance to Races }\end{array}$ & $\begin{array}{l}\text { Institution and Location/Country } \\
\text { Where Germplasm Was Screened }\end{array}$ & $\begin{array}{l}\text { Known Use to Mitigate } \\
\text { Fusarium Impact }\end{array}$ & References \\
\hline M. ruiliensis & * & TR4 & $\mathrm{R}$ & $\begin{array}{l}\text { South China Agricultural } \\
\text { University }\end{array}$ & Wild germplasm & [75] \\
\hline M. velutina & * & TR4 & $\mathrm{R}$ & $\begin{array}{c}\text { South China Agricultural } \\
\text { University }\end{array}$ & Wild germplasm & [75] \\
\hline Nantianqing & AAA & TR4 & MR & $\begin{array}{l}\text { Dongguan Banana Vegetable } \\
\text { Institute, China }\end{array}$ & China & [51] \\
\hline Dongjiao 1 & AAA & TR4 & MR & $\begin{array}{l}\text { Dongguan Banana Vegetable } \\
\text { Institute, China }\end{array}$ & China & [51] \\
\hline Kangku 1 & AAA & TR4 & $\mathrm{R}$ & $\begin{array}{l}\text { Dongguan Banana Vegetable } \\
\text { Institute, China }\end{array}$ & China & [51] \\
\hline G6-2 & AAA & TR4 & $\mathrm{R}$ & $\begin{array}{l}\text { Dongguan Banana Vegetable } \\
\text { Institute, China }\end{array}$ & China & [51] \\
\hline Yueke 1 & AAA & TR4 & MR & $\begin{array}{l}\text { Dongguan Banana Vegetable } \\
\text { Institute, China }\end{array}$ & China & [51] \\
\hline Nongke 1 & AAA & TR4 & MR & $\begin{array}{l}\text { Dongguan Banana Vegetable } \\
\text { Institute, China }\end{array}$ & China & [51] \\
\hline Kangku 5 & AAA & TR4 & HR & $\begin{array}{l}\text { Dongguan Banana Vegetable } \\
\text { Institute, China }\end{array}$ & China & [51] \\
\hline Nantianhuang & AAA & TR4 & MR & $\begin{array}{l}\text { Dongguan Banana Vegetable } \\
\text { Institute, China }\end{array}$ & China & [51] \\
\hline BXM51 & AAA & TR4 & MR & $\begin{array}{l}\text { Dongguan Banana Vegetable } \\
\text { Institute, China }\end{array}$ & China & [51] \\
\hline $\begin{array}{c}\text { Yueyoukang } \\
1\end{array}$ & AAA & TR4 & $\mathrm{R}$ & $\begin{array}{l}\text { South China Agricultural } \\
\text { University }\end{array}$ & China & [113] \\
\hline $\begin{array}{l}\text { Pisang Gajih } \\
\text { Merah }\end{array}$ & AAA & TR4 & SS & $\begin{array}{l}\text { University of Queensland, } \\
\text { Australia }\end{array}$ & Australia & [72] \\
\hline $\begin{array}{l}\text { GCTCV-218 } \\
\text { Formosana }\end{array}$ & AAA & TR4 & $\mathrm{R}$ & $\begin{array}{c}\text { University of Queensland, } \\
\text { Australia and Northern } \\
\text { Mozambique }\end{array}$ & $\begin{array}{l}\text { China, Taiwan, } \\
\text { Philippines and } \\
\text { Mozambique. }\end{array}$ & {$[5,72]$} \\
\hline $\begin{array}{l}\text { FHIA-01 } \\
\text { (“Goldfin- } \\
\text { ger") }\end{array}$ & AAAB & $\begin{array}{l}\text { Race } \\
\text { 1/STR4 }\end{array}$ & $\mathrm{R}$ & $\begin{array}{l}\text { DAFF, Australia [84], FHIA, } \\
\text { Honduras [93] }\end{array}$ & $\begin{array}{l}\text { Africa, Australia, } \\
\text { Honduras }\end{array}$ & {$[84,93]$} \\
\hline Tuu Gia & AA & $\begin{array}{l}\text { Race } \\
1 / \text { TR4 }\end{array}$ & HR & $\begin{array}{l}\text { (IFTR-GDAAS), } \\
\text { Guangzhou, China }\end{array}$ & In breeding programs & [77] \\
\hline Pisang Lilin & AA & $\begin{array}{l}\text { Race } \\
1 / \text { TR4 }\end{array}$ & $\mathrm{R}$ & $\begin{array}{l}\text { (IFTR-GDAAS), } \\
\text { Guangzhou, China }\end{array}$ & In breeding programs & [77] \\
\hline Borneo & AA & $\begin{array}{l}\text { Race } \\
\text { 1/TR4 }\end{array}$ & $\mathrm{R}$ & $\begin{array}{c}\text { National Agricultural Research } \\
\text { Laboratories (NARL) [31] } \\
\text { (IFTR-GDAAS), } \\
\text { Guangzhou, China [80] and } \\
\text { Wageningen University and Research, } \\
\text { Wageningen, Netherlands [102] }\end{array}$ & In breeding programs & {$[31,77,102]$} \\
\hline $\begin{array}{l}\text { Pisang } \\
\text { Berlin }\end{array}$ & AA & $\begin{array}{l}\text { Race } \\
1 / \text { TR4 }\end{array}$ & $\mathrm{R}$ & $\begin{array}{c}\text { (IFTR-GDAAS), } \\
\text { Guangzhou, China [77], Embrapa } \\
\text { cassava and fruit growing, Brazil [78] }\end{array}$ & In breeding programs & {$[77,78]$} \\
\hline Zebrina GF & * & $\begin{array}{l}\text { Race } \\
1 / \text { TR4 }\end{array}$ & $\mathrm{R}$ & $\begin{array}{c}\text { University of Malaya, Kuala } \\
\text { Lumpur, Malaysia [31], } \\
\text { IFTR-GDAAS, } \\
\text { Guangzhou, China [77] }\end{array}$ & In breeding programs & {$[31,77]$} \\
\hline
\end{tabular}


Table 4. Cont.

\begin{tabular}{|c|c|c|c|c|c|c|}
\hline $\begin{array}{c}\text { Musa } \\
\text { Germplasm }\end{array}$ & $\begin{array}{l}\text { Musa } \\
\text { Genome }\end{array}$ & $\begin{array}{l}\text { Lev } \\
\text { Re }\end{array}$ & $\begin{array}{l}\text { evel of Tolerance or } \\
\text { Resistance to Races }\end{array}$ & $\begin{array}{l}\text { Institution and Location/Country } \\
\text { Where Germplasm Was Screened }\end{array}$ & $\begin{array}{l}\text { Known Use to Mitigate } \\
\text { Fusarium Impact }\end{array}$ & References \\
\hline Pahang & AA & $\begin{array}{l}\text { Race } \\
\text { 1/STR4/TR4 }\end{array}$ & $\mathrm{HR}$ & $\begin{array}{c}\text { University of Queensland, } \\
\text { Australia [72], Yunnan Agricultural } \\
\text { University, } \\
\text { Kunming, China [74,114] and } \\
\text { IFTR-GDAAS, } \\
\text { Guangzhou, China [77] }\end{array}$ & In breeding programs & $\begin{array}{c}{[72,74,77} \\
114]\end{array}$ \\
\hline Calcutta-4 & AA & $\begin{array}{l}\text { Race } \\
\text { 1/STR4/TR4 }\end{array}$ & HR & $\begin{array}{c}\text { University of Queensland, } \\
\text { Australia [66] and (IFTR-GDAAS), } \\
\text { Guangzhou, China [72] }\end{array}$ & In breeding programs & {$[72,77]$} \\
\hline Ma851 & AA & STR4/TR4 & $\mathrm{R}$ & $\begin{array}{l}\text { University of Queensland, } \\
\text { Australia }\end{array}$ & In breeding programs & [72] \\
\hline Ma852 & AA & STR4/TR4 & $\mathrm{R}$ & $\begin{array}{l}\text { University of Queensland, } \\
\text { Australia }\end{array}$ & In breeding programs & [72] \\
\hline $\begin{array}{l}\text { Calcutta4- } \\
\text { IV9 }\end{array}$ & AA & STR4/TR4 & $\mathrm{R}$ & $\begin{array}{c}\text { University of Queensland, } \\
\text { Australia [66] and IFTR-GDAAS, } \\
\text { Guangzhou, China [72] }\end{array}$ & In breeding programs & {$[72,77]$} \\
\hline SH-3362 & AA & STR4/TR4 & $\mathrm{R}$ & $\begin{array}{l}\text { University of Queensland, } \\
\text { Australia }\end{array}$ & In breeding programs & [72] \\
\hline SH-3142 & AA & STR4/TR4 & $\mathrm{R}$ & $\begin{array}{l}\text { University of Queensland, } \\
\text { Australia }\end{array}$ & In breeding programs & {$[72]$} \\
\hline $\begin{array}{c}\text { Madang } \\
\text { Guadeloupe }\end{array}$ & AA & STR4/TR4 & $\mathrm{R}$ & $\begin{array}{l}\text { University of Queensland, } \\
\text { Australia }\end{array}$ & In breeding programs of & [72] \\
\hline $\begin{array}{l}\text { FHIA-1 } \\
\text { ("Gold } \\
\text { Finger") }\end{array}$ & AAAB & STR4/TR4 & $\mathrm{R}$ & $\begin{array}{l}\text { University of Queensland, } \\
\text { Australia }\end{array}$ & $\begin{array}{c}\text { Australia, Brazil, } \\
\text { Mexico, Colombia, EUA }\end{array}$ & [72] \\
\hline FHIA-25 & $\mathrm{AAB}$ & STR4/TR4 & $\mathrm{R}$ & $\begin{array}{c}\text { University of Queensland, } \\
\text { Australia [72], (IFTR-GDAAS), } \\
\text { Guangzhou, China [77], } \\
\text { Wageningen University and } \\
\text { Research, Wageningen, } \\
\text { Netherlands [102] }\end{array}$ & $\begin{array}{l}\text { Africa, Latin America } \\
\text { and Australia } \\
\text { (Honduras, Colombia, } \\
\text { Brazil, Jamaica, } \\
\text { Mozambique) }\end{array}$ & {$[72,77,102]$} \\
\hline GCTCV-119 & AAA & STR4/TR4 & $\mathrm{R}$ & $\begin{array}{l}\text { University of Queensland, } \\
\text { Australia and Northern } \\
\text { Mozambique }\end{array}$ & $\begin{array}{l}\text { China, Taiwan, The } \\
\text { Philippines, } \\
\text { Mozambique }\end{array}$ & {$[5,72]$} \\
\hline Ma850 & AA & ST4/TR4 & $\mathrm{R}$ & $\begin{array}{l}\text { University of Queensland, } \\
\text { Australia }\end{array}$ & In breeding programs & [72] \\
\hline $\begin{array}{l}\text { Pisang Jari } \\
\text { Buaya }\end{array}$ & AA & STR4/TR4 & $\mathrm{R}$ & $\begin{array}{c}\text { University of Queensland, } \\
\text { Australia [72] and (IFTR-GDAAS), } \\
\text { Guangzhou, China [77] }\end{array}$ & In breeding programs & {$[72,77]$} \\
\hline FHIA-18 & $\mathrm{AAAB}$ & STR4/TR4 & $\mathrm{R}$ & $\begin{array}{c}\text { University of Queensland, } \\
\text { Australia [72], IFTM Brazil [81], } \\
\text { DAFF, Australia [84], Federal } \\
\text { University of Santa Catarina, Brazil } \\
\text { [111] }\end{array}$ & $\begin{array}{l}\text { Africa, Latin America } \\
\text { and Australia } \\
\text { (Honduras, Colombia, } \\
\text { Jamaica, Mozambique) }\end{array}$ & $\begin{array}{l}{[72,81,84} \\
111]\end{array}$ \\
\hline
\end{tabular}

R, SS, MS, S, and HS abbreviate resistant, slightly susceptible, moderately susceptible, susceptible, and highly susceptible. EAHBs = East African Highland Bananas; IFTR-GDAAS = Institute of Fruit Tree Research, Guangdong Academy of Agricultural Sciences; EMBRAPA = Brazilian agricultural research corporation; DAFF = Department of Agriculture, Fisheries and Forestry. 


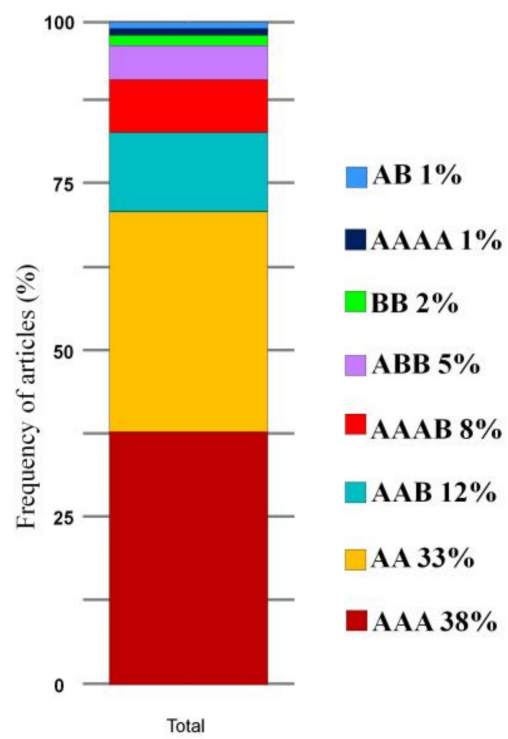

Figure 8. Frequency of genomes associated with sources of resistance to Fusarium wilt in studies on banana breeding carried out in the last ten years.

The resistance sources reported that are exclusively related to FOC TR4 included the diploid cultivars, Pahang, Calcutta-4, Zebrina, Pisang Lilin, Malaccensis, Jari Buaya, and Tuu Gia, all with a higher frequency, according to the word cloud (Figure 9A). Besides these, other cultivars have also been reported as resistant to FOC TR4 in field tests, such as the hybrids FHIA-01, FHIA-02, SH-3748, SH-3362, FHIA-25, SH-3142, and SH-3362 (Figure 9A). According to genome frequency data related to resistance sources, most genotypes reported as resistant to FOC TR4 are AA diploid genomes (45\%), AAA triploid genomes (21\%), and AAB triploid genomes (18\%) (Figure 9B).

a)

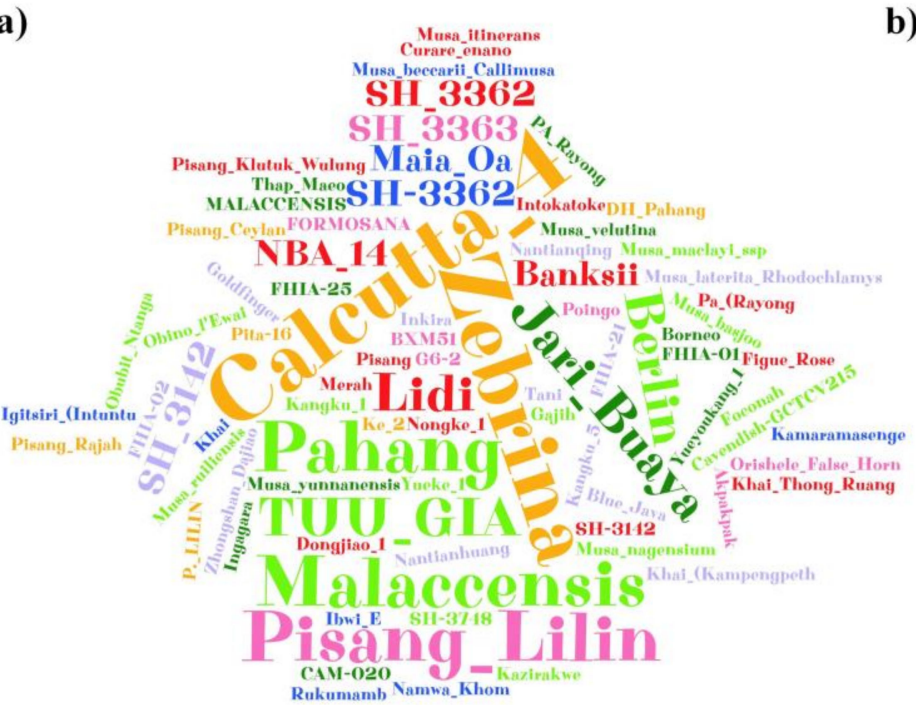

b)

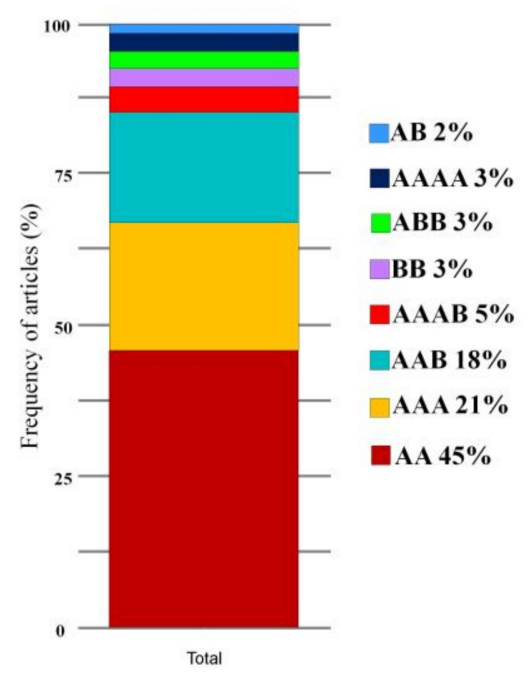

Figure 9. Sources of resistance to Fusarium oxysporum f. sp. cubense tropical race 4 (TR4) in studies on the improvement of banana plants to Fusarium wilt in the last ten years. (a) Word cloud of the frequency of cited sources of resistance. (b) Frequency of genomes related to the sources of resistance cited. 


\subsection{Gene Expression Analysis}

Figure 10A shows the gene categories present in studies on gene analysis and expression. The highest frequency of articles found genes associated with pathogenesis and defense (57\%) (Figure 10A). Other genes, studied at a lower frequency, are related to RNAs $(12 \%)$, hormone biosynthesis $(10 \%)$, kinases $(9 \%)$, transcription factors $(6 \%)$, genes related to autophagy (4\%), and starch biosynthesis ( $2 \%)$. A summary of the main genes related to each category can be found in Table S1.

a)

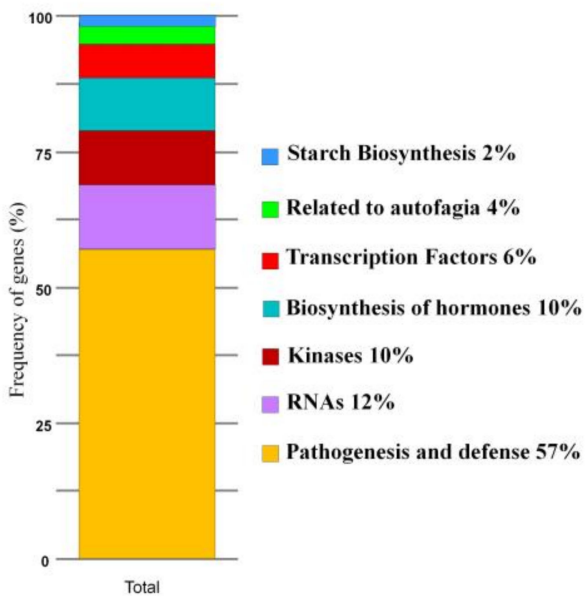

b)

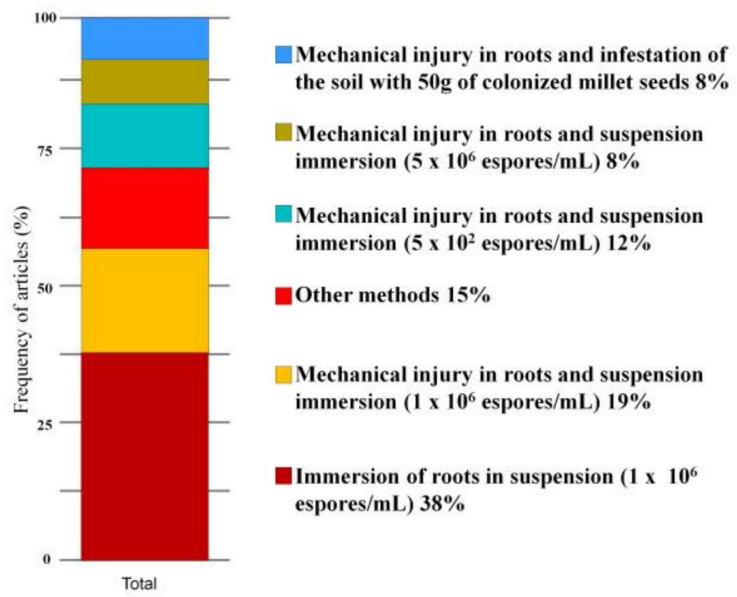

Figure 10. Gene expression studies of banana plants infected with Fusarium oxysporum $\mathrm{f}$. sp. cubense in articles carried out in the last ten years. Categories of genes associated with the frequency of articles (a) and frequency of methods used for inoculation of plants to check gene expression (b).

Methods for host plant inoculation to analyze gene expression after FOC infection are not standardized among the analyzed articles $(n=27)$, with several methods adopted (Figure 10B). The highest frequency of articles related to the inoculation method with conidia suspension at a concentration of $1 \times 10^{6}$ spores $\mathrm{mL}^{-1}(38 \%)$, followed by the inoculation method by mechanical damage to the roots and then immersion in suspension at a concentration of $1 \times 10^{6} \mathrm{~mL}^{-1}$ spores $(19 \%)$. Other methods that were present in a single article represented 15\% cumulatively (Figure 10B). The method of mechanical root damage and immersion in suspension at a concentration of $5 \times 10^{2}$ spores $\mathrm{mL}^{-1}$ represented $12 \%$ of the articles and the methods of mechanical root damage and immersion in suspension at a concentration of $5 \times 10^{6}$ spores $\mathrm{mL}^{-1}$ and mechanical root damage and soil infestation with $50 \mathrm{~g}$ of colonized millet seeds represented $8 \%$ of the articles (Figure 10B). Therefore, we observed that the main differences were related to whether the roots were wounded and the spore concentration adopted in each case regarding the inoculation method.

Table 5 is from the study by Wang et al. [115], which was modified, to show all the selected articles that evaluated the banana transcriptome infected by FOC TR4 and FOC R1. These studies observed the changes in expression of defense-related genes related to different enriched pathways, from gene annotation pathways, namely Gene Ontology (GO) annotation and the Kyoto encyclopedia of genes and genomes-based pathway analysis (KEGG-PATH). According to most transcriptome studies, the pathways activated after FOC infection were related to phenylpropanoid biosynthesis, sugar biosynthesis, cell wall modifications, flavonoid biosynthesis, and plant hormone signal transduction (Table 5). The main genes related to the above-mentioned pathways are listed in Table S1. 
Table 5. Transcriptomic studies involving banana plants infected with Fusarium oxysporum $\mathrm{f}$. sp. cubense in articles about the improvement of banana to Fusarium wilt, carried out in the last ten years *.

\begin{tabular}{|c|c|c|c|c|c|}
\hline Article & Banana Variety & $\begin{array}{l}\text { Plant Growth } \\
\text { Stage }\end{array}$ & Race & $\begin{array}{l}\text { Sampling (after } \\
\text { Infection) }\end{array}$ & $\begin{array}{l}\text { Pathways Enriched for Differentially } \\
\text { Expressed Genes }\end{array}$ \\
\hline Wang et al. [115] & $\begin{array}{l}\text { Banana "Brazil" } \\
\text { (susceptible) and } \\
\text { "Formosana" } \\
\text { (tolerant) }\end{array}$ & 4.5 months & FOC TR4 & $48 \mathrm{~h}$ & $\begin{array}{l}\text { Flavonoid biosynthesis, flavone and flavonol } \\
\text { biosynthesis, alpha-linolenic acid metabolism, } \\
\text { starch and sucrose metabolism and } \\
\text { phenylpropanoid biosynthesis. }\end{array}$ \\
\hline Wang et al. [116] & Banana "Brazil" & $60 \mathrm{~d}$ & FOCTR4 & $0,2,4,6$ days & $\begin{array}{l}\text { Phenylalanine metabolism, phenylpropanoid } \\
\text { biosynthesis, drug metabolism-cytochrome } \\
\text { P450, alpha-linolenic acid metabolism, amino } \\
\text { sugar and nucleotide sugar metabolism. }\end{array}$ \\
\hline Li et al. [37] & Banana "Brazil" & $50 \mathrm{~d}$ & $\begin{array}{l}\text { FOC } 1 \text { and } \\
\text { FOC TR4 }\end{array}$ & $3,27,51 \mathrm{~h}$ & $\begin{array}{l}\text { PR proteins, phytoalexins and } \\
\text { phenylpropanoid synthesis, cell wall } \\
\text { modifications, biosynthesis via } \\
\text { ethylene signaling. }\end{array}$ \\
\hline Li et al. [117] & $\begin{array}{l}\text { Banana "Brazil" } \\
\text { and "Nongke No } 1 " \\
\text { (resistant) }\end{array}$ & $\begin{array}{l}\text { Plants with four } \\
\text { or five leaves }\end{array}$ & FOC TR4 & $48,96 \mathrm{~h}$ & $\begin{array}{l}\text { Perception of PAMP by PRRs, hormone } \\
\text { biosynthesis and signaling, transcription } \\
\text { factors, cell wall modification, flavonoid } \\
\text { biosynthesis, programmed cell death, } \\
\text { PR proteins }\end{array}$ \\
\hline Bai et al. [113] & $\begin{array}{l}\text { Banana "Brazil" } \\
\text { and "Yueyoukang } \\
1 \text { " resistant }\end{array}$ & $\begin{array}{l}8 \text { weeks (plants } \\
\text { with five leaves) }\end{array}$ & FOC TR4 & $\begin{array}{l}0,5,1,3,5,10 \\
\quad \text { days }\end{array}$ & $\begin{array}{l}\text { PR proteins, transcription factors, cell wall } \\
\text { modification, phenylpropanoid biosynthesis, } \\
\text { plant hormone signal transduction. }\end{array}$ \\
\hline Zhang et al. [114] & $\begin{array}{l}\text { Musa acuminata } \\
\text { Pahang and } \\
\text { Brazilian }\end{array}$ & & FOC STR4 & at 14 days & $\begin{array}{l}\text { PR proteins, transcription factors, cell wall } \\
\text { modification, phenylpropanoid biosynthesis, } \\
\text { plant hormone signal transduction. }\end{array}$ \\
\hline Sun et al. [32] & $\begin{array}{l}\text { Musa acuminata " } \\
\text { Guijiao } 9^{\prime \prime} \text { and } \\
\text { Williams }\end{array}$ & 6 months & FOC TR4 & At 6 days & $\begin{array}{l}\text { Membrane-bound intracellular organelle, cell } \\
\text { wall and cytoplasm, ions, transcription factor } \\
\text { and oxidoreductase activity, plant-pathogen } \\
\text { interaction, plant hormone signal transduction, } \\
\text { phenylpropanoid biosynthesis and } \\
\text { flavonoid biosynthesis. }\end{array}$ \\
\hline Fei et al. [118] & Cavendish banana & 3 months & $\begin{array}{l}\text { FOC } 1 \text { and } \\
\text { FOC TR4 }\end{array}$ & At 28 days & $\begin{array}{l}\text { Cell components, molecular function and } \\
\text { biological process. }\end{array}$ \\
\hline Cheng et al. [103] & $\begin{array}{l}\text { Musa acuminata cv. } \\
\text { Tianbaojiao }\end{array}$ & 11 weeks & FOC TR4 & $5,10,25 \mathrm{~h}$ & $\begin{array}{l}\text { Auxin-activated signaling pathway, cellular } \\
\text { response, auxin stimulation, phenylpropanoid } \\
\text { catabolic process, lignin catabolic process, } \\
\text { lignin metabolic process, via peroxisomes. }\end{array}$ \\
\hline Song et al. [119] & $\begin{array}{l}\text { Brazilian banana } \\
\text { and señorita } \\
\text { banana }\end{array}$ & $\begin{array}{l}\text { In the five-leaf } \\
\text { stage }\end{array}$ & $\begin{array}{l}\text { FOC } 1 \text { and } \\
\text { FOC TR4 }\end{array}$ & $\begin{array}{l}\text { In the five-leaf } \\
\text { stage }\end{array}$ & $\begin{array}{l}\text { Cellular process, metabolic process and } \\
\text { binding of organelles and nucleic acids or } \\
\text { proteins, regulation of biological processes and } \\
\text { transcription factors. }\end{array}$ \\
\hline Li et al. [120] & $\begin{array}{l}\text { Cavendish banana } \\
\text { and Brazilian (BX) }\end{array}$ & 90 days & FOC TR4 & $27 \mathrm{~h}, 51 \mathrm{~h}$ & $\begin{array}{l}\text { Secondary metabolite biosynthesis, } \\
\text { plant-pathogen interaction, phenylpropanoid } \\
\text { biosynthesis and phenylalanine metabolism, } \\
\text { fatty acid metabolism, glycerolipid and } \\
\text { glycerophospholipid metabolism }\end{array}$ \\
\hline Niu et al. [121] & $\begin{array}{l}\text { Yueyoukang1 and } \\
\text { Baxijiao }\end{array}$ & 2 weeks & FOC TR4 & $24 \mathrm{~h}$ & $\begin{array}{l}\text { Cell wall biosynthesis and degradation, cell } \\
\text { polysaccharide metabolic process, chitinase } \\
\text { activity, pectinesterase activity and xyloglucan } \\
\text { activity, fructose and mannose metabolism, } \\
\text { sphingolipid metabolism, butanoate } \\
\text { metabolism, porphyrin and chlorophyll } \\
\text { metabolism, carotenoid and } \\
\text { ribosome biosynthesis. }\end{array}$ \\
\hline
\end{tabular}

\footnotetext{
* modified table by Wang et al. [115].
} 
3.6. Studies on the Achievement and Evaluation of Hybrids and on Genetic Inheritance of Musa spp.

The studies related to crossbreeding to obtain resistant hybrids or those focused on evaluating the genetic inheritance in Musa spp., as well as the parental lineages used and their genealogies, are listed in Table 6. Ssali et al. [93] produced hybrids from crossbreeding the resistant diploid TMB2X8075 (originated from the cross between SH3362 (AA) and Calcutta 4 (AA)) and Sukali Ndizi (AAB), which is also resistant to FOC R1 and 4, to evaluate the inheritance of the resistance of Musa spp. to FOC R1 in three F2 populations. Concerning the progeny, the authors found that 115 were susceptible, and 48 were resistant. Similarly, in the study by Arinaitwe et al. [31], crossbreeding between Monyet (Musa acuminata ssp. Zebrina) and Kokopo (Musa acuminata ssp. Banksii) were performed to identify suitable banana germplasm to generate a segregating F1 population and to understand the mode of inheritance of resistance to FOC R1 (Table 6).

Table 6. Evaluation of hybrids and genetic inheritance studies in articles about the improvement of banana plants to fusarium wilt carried out in the last ten years.

\begin{tabular}{|c|c|}
\hline Hybrids & Parentage \\
\hline \multicolumn{2}{|r|}{ Article } \\
\hline \multicolumn{2}{|r|}{ Ssali et al. [93] } \\
\hline F2 progenies & Diploid TMB2X8075 (“SH3362" (AA) × “Calcutta 4" (AA) $\times$ Sukali Ndizi (AAB) \\
\hline \multicolumn{2}{|r|}{ Arinaitwe et al. [31] } \\
\hline F1 progenies & Monyet (Musa acuminata ssp. Zebrina) $\times$ Kokopo (Musa acuminata ssp. Banksii) \\
\hline \multicolumn{2}{|r|}{ Ahmad et al. [108] } \\
\hline & Musa acuminata ssp. Malaccensis (selfed) \\
\hline \multicolumn{2}{|r|}{ Gonçalves et al. [33] } \\
\hline CNPMF0038 & $(($ M53 $\times$ Madu $)) \times(($ Malaccensis $\times$ Tjau Lagada $))$ \\
\hline CNPMF0496 & $(($ M61 $\times$ Pisang Lilin $)) \times(($ Terrinha $\times$ Calcutta 4$))$ \\
\hline CNPMF0513 & $(($ M61 $\times$ Pisang Lilin $)) \times(($ M53 $\times$ Kumburgh $))$ \\
\hline CNPMF0519 & Self-fertilization (wild diploid Tambi) \\
\hline CNPMF0534 & $(($ Calcutta $4 \times$ Madang $)) \times(($ Borneo $\times$ Guyod $))$ \\
\hline CNPMF0536 & $(($ Calcutta $4 \times$ Madang $)) \times(($ Borneo $\times$ Guyod $))$ \\
\hline CNPMF0542 & $((\mathrm{SH} 3263)) \times(($ Malaccensis $\times$ Sinwobogi $))$ \\
\hline CNPMF0557 & $(($ M61 $\times$ Pisang Lilin $)) \times(($ Malaccensis $\times$ Tjau Lagada $))$ \\
\hline CNPMF0565 & $(($ Calcutta $4 \times$ Pahang $) \times($ Borneo $\times$ Madang $)) \times$ Khae \\
\hline CNPMF0572 & $(($ Khai $\times($ Calcutta $4 \times$ Madang $)) \times(($ Calcutta $4 \times$ Madang $))$ \\
\hline CNPMF0612 & $(($ M53 $\times$ Madu $) \times$ Madu $)) \times$ SH3263 \\
\hline CNPMF0731 & $(($ Malaccensis $\times$ Madang $)) \times(($ Tuugia $\times$ Calcutta 4$))$ \\
\hline CNPMF0767 & $(($ Malaccensis $\times$ Madang $)) \times(($ Khai $\times($ Calcutta $4 \times$ Madang $))$ \\
\hline CNPMF0811 & $(($ Khai $\times($ Calcutta $4 \times$ Madang $)) \times(($ Calcutta $4 \times$ Pahang $) \times($ Borneo $\times$ Madang $))$ \\
\hline CNPMF0978 & $(($ Calcutta $4 \times$ Madang $)) \times(($ Terrinha $\times$ Calcutta 4$))$ \\
\hline CNPMF0993 & $(($ Borneo $\times$ Guyod $) \times($ Tuugia $\times$ Calcutta 4$)) \times(($ Khai $\times($ Calcutta $4 \times$ Madang $))$ \\
\hline CNPMF0998 & $(($ Borneo $\times$ Guyod $)) \times(($ Borneo $\times$ Guyod $) \times \mathrm{SH} 3263)$ \\
\hline CNPMF1102 & $($ (Jari Buaya $\times($ Calcutta $4 \times$ Madang $)) \times(($ Borneo $\times$ Guyod $) \times($ Tuugia $\times$ Calcutta 4$))$ \\
\hline CNPMF1105 & $(($ Borneo $\times$ Guyod $) \times($ Calcutta $4 \times$ Heva $)) \times(($ Calcutta $4 \times$ Madang $))$ \\
\hline CNPMF1171 & $(($ Malaccensis $\times$ Madang $)) \times(($ M53 $\times($ Tuugia $\times$ Calcutta 4$))$ \\
\hline CNPMF1272 & $(($ Borneo $\times$ Guyod $) \times($ Calcutta $4 \times$ Heva $)) \times(($ Tuugia $\times$ Calcutta 4$))$ \\
\hline CNPMF1286 & $(($ Calcutta $4 \times$ Madang $)) \times(($ Terrinha $\times$ Calcutta 4$))$ \\
\hline
\end{tabular}


Table 6. Cont.

\begin{tabular}{lc}
\hline \multicolumn{1}{c}{ Hybrids } & Parentage \\
\hline CNPMF1323 & $(($ Malaccensis $\times$ Sinwobogi $)) \times(($ Calcutta $4 \times$ Heva $))$ \\
\hline CNPMF0241 & $(($ Pacovan $\times$ improved diploid $))$ \\
\hline CNPMF0282 & $(($ Pacovan $\times$ improved diploid $))$ \\
\hline CNPMF0351 & $(($ Prata Anã $\times$ improved diploid by FHIA $))$ \\
\hline CNPMF0897 & $(($ Prata Anã $)) \times(($ Malaccensis $\times$ Sinwobogi $) \times($ Zebrina $\times$ Heva $))$ \\
\hline CNPMF0898 & $(($ Prata Anã $)) \times(($ Malaccensis $\times$ Sinwobogi $) \times($ Calcutta $4 \times$ Galeo $))$ \\
\hline CNPMF0906 & $(($ Prata Santa Maria $\times$ improved diploid $))$ \\
\hline CNPMF0908 & $(($ Silk $\times$ improved diploid $))$ \\
\hline BRS Princesa & $(($ Yangambi $\times$ M53 $))$ \\
\hline
\end{tabular}

The study by Ahmad et al. [108] is the first report of the genetic basis of resistance to FOC R1 in bananas using heterozygous wild banana Musa acuminata ssp. malaccensis (AA) to generate a mapping population and investigate the inheritance of resistance to FOC R1 and FOC TR4 through genetic mapping. This study demonstrated that resistance to FOC $\mathrm{R} 1$ is inherited as a single gene and that M. acuminata ssp. malaccensis is fertile and can be a potential parent to create resistance to Fusarium wilt.

Among the hybrids studied by Gonçalvez et al. [33], the improved diploids (CNPMF0038, CNPMF0513, CNPMF0767, and CNPMF1171) and the tetraploid hybrid BRS Princesa were considered moderately resistant (Table 6). All other hybrids evaluated in their study were considered resistant to Fusarium wilt caused by FOC R1. Gonçalvez et al. [33] mostly used improved male diploid parents, resulting from crossbreeding with diploids resistant to FOC R1 and FOC TR4, such as Calcutta 4 and M53.

\subsection{Transgenesis}

In the articles reporting the use of transgenesis $(n=14)$, the tool for genetic transformation was mediated by Agrobacterium tumefaciens, using embryogenic cell suspension culture. One exception is a method proposed by Subramaniam et al. [122], who, in addition to agroinoculation, developed a biolistics method. In this study, we used a table developed by Poon and Teo [123] as a model to show information about the works of this systematic review related to transgenesis (Table 7). In Table 7, we observed that most studies used the Rasthali cultivar (AAB) for genetic engineering. 
Table 7. Genes used transgenics in studies on genetic improvement of banana to Fusarium wilt in the last ten years.

\begin{tabular}{|c|c|c|c|c|}
\hline Gene & Sources & Function & Banana Cultivar & References \\
\hline $\begin{array}{l}\text { Ferredoxin (Atfd3) and } \\
\text { ferredoxin-like protein } \\
\text { (pflp) }\end{array}$ & Capsicum annuum & Antimicrobial peptide & cv. Pei Chiao (AAA) & [69] \\
\hline $\begin{array}{l}\text { Petunia floral defenses } \\
\text { (PhDef1 and PhDef2) }\end{array}$ & Petunia hybrida & Antimicrobial peptide & cv. Rasthali (AAB) & [124] \\
\hline Onion-Ace-AMP1 & Allium cepa & Antimicrobial peptide & cv. Rasthali (AAB) & {$[88]$} \\
\hline Endochitinase (chit42) & Trichodermaharzianum & Antifungal activity & cv. Furenzhi (AA) & [125] \\
\hline Defensin (Sm-AMP-D1) & Stellaria media & Antimicrobial peptide & cv. Rasthali (AAB) & [126] \\
\hline $\begin{array}{c}\text { Small interfering } \\
\text { RNAs(siRNAs)/(ihpRNA) }\end{array}$ & - & Silencing of vital fungal genes & cv. Rasthali (AAB) & [83] \\
\hline $\begin{array}{c}\text { (MusaDAD1, } \\
\text { MusaBAG1 eMusaBI1) }\end{array}$ & Musa acuminata & $\begin{array}{l}\text { Cell death is highly induced by } \\
\text { FOC infection }\end{array}$ & cv. Rasthali (AAB) & [96] \\
\hline $\begin{array}{l}\text { Cell death (Bcl-Xl, } \\
\text { Ced-9 e Bcl-23) }\end{array}$ & Caenorhabditiselegans & Antiapoptosis & cv. Grand Naine & [89] \\
\hline Cell death (Ced9) & Caenorhabditiselegans & Antiapoptosis & $\begin{array}{l}\text { cv. Sukali Ndizi (Musa } \\
\text { ssp. AAB) }\end{array}$ & [100] \\
\hline $\begin{array}{l}\text { Pathogenesis-reported } \\
\text { (MaPR-10) }\end{array}$ & $\begin{array}{l}\text { Musa acuminata ssp. } \\
\text { malaccensis }\end{array}$ & Pathogenesis (PR) & $\begin{array}{l}\text { M. acuminata cv. } \\
\text { Berangan }\end{array}$ & [73] \\
\hline (RGA2) and (Ced9) & $\begin{array}{l}\text { Musa acuminata ssp. } \\
\text { malaccensis } \\
\text { /Caenorhabditis elegans }\end{array}$ & Resistance analog/antiapoptosis & cv. Grand Naine & [127] \\
\hline $\begin{array}{l}\text { Chitinases and } \\
\text { 1.3-glucanase }\end{array}$ & Oryza sativa & Disease tolerance & cv. Rasthali (AAB) & [122] \\
\hline $\begin{array}{l}\text { Synthesis of ergosterol } \\
\text { (ERG6) }\end{array}$ & - & Silencing of vital fungal genes & Cavendish & [128] \\
\hline $\begin{array}{l}\text { Small interfering } \\
\text { RNAs-ihpRNA }\end{array}$ & - & Silencing of vital fungal genes & cv. Rasthali (AAB) & [129] \\
\hline
\end{tabular}

Among the genes used for transgenesis, there was a considerable frequency of studies using transgenes as the antiapoptosis gene (Ced9) from the nematode Caenorhabditis elegans (Table 7). Two genes derived from Musa acuminata ssp. malaccensis, one related to pathogenesis (MaPR-10) and the other a resistance analog (RGA2), were also successfully used in this case as cisgenes. Other cell death genes, MusaDAD1, MusaBAG1, and MusaBI1, from Musa acuminata were also efficient, particularly MusaBAG1. In addition to these, the RNA interference technology enables the silencing of vital genes of FOC when employing small interfering RNA (siRNA) and intron-containing hairpin RNA (ihpRNA) (Table 7).

Four antimicrobial peptides from the plant species Capsicum annuum, Petunia hybrida, Allium cepa, and Stellaria media and an antifungal activity gene from Trichoderma harzianum were also successfully used to obtain resistant transgenic banana plants (Table 7).

\subsection{Induction of Resistance}

Among the inducers, the biocontrol agents Bacillus subtilis, Trichoderma spp., and Penicillium citrinum were the most reported for exploring induction of systemic resistance (Table 8). Chemical induction agents were also reported, such as the plant hormones abscisic acid (ABA), methyl jasmonate (MeJA), and salicylic acid (SA), in addition to benzothiadiazole (BTH). Other studies explored induced systemic resistance with the FOC pathogen in different ways (Table 8). 
Table 8. Resistance-inducing agents in banana plants reported in studies on improvement to Fusarium wilt in the last ten years.

\begin{tabular}{|c|c|c|}
\hline Inductor & Application & References \\
\hline Bacillus subtilis & $\begin{array}{l}\text { Inoculation of plants with suspension } \\
\text { in a greenhouse }\end{array}$ & [130] \\
\hline Trichoderma asperellum & $\begin{array}{l}\text { Inoculation of plants with suspension } \\
\text { in a greenhouse }\end{array}$ & [131] \\
\hline $\begin{array}{l}\text { Abscisic acid (ABA), ethephon, } \\
\text { methyl jasmonate (MeJA) and } \\
\text { salicylic acid (SA) }\end{array}$ & Root treatment with inductor solutions & [132] \\
\hline Penicillium citrinum & $\begin{array}{l}\text { Inoculation of plants with suspension } \\
\text { in a greenhouse }\end{array}$ & [88] \\
\hline Bacillus subtilis & $\begin{array}{l}\text { Treatment with in vitro fermented } \\
\text { culture filtrate and inoculation of plants } \\
\text { with suspension in a greenhouse }\end{array}$ & {$[90]$} \\
\hline Benzothiadiazole (BTH) & Spraying leaves and roots & [133] \\
\hline Interaction with dead FOC pathogen & $\begin{array}{l}\text { Inoculation of plants with suspension } \\
\text { in a greenhouse }\end{array}$ & [134] \\
\hline Methyl jasmonate (MeJA) & $\begin{array}{l}\text { Exogenous solution treatment in soil } \\
\text { and leaves }\end{array}$ & [76] \\
\hline $\begin{array}{c}\text { A strain of FOC } 1 \text { incompatible with } \\
\text { inducing resistance against the } \\
\text { tropical race } 4 \text { TR } 4\end{array}$ & $\begin{array}{l}\text { Systemic resistance acquired by in vitro } \\
\text { inoculation }\end{array}$ & [99] \\
\hline $\begin{array}{l}\text { Isolates of Trichoderma spp. ( } T \text {. } \\
\text { koningii, T. viride, T. harzianum) }\end{array}$ & $\begin{array}{c}\text { Biomass, liquid culture and culture } \\
\text { filtrate }\end{array}$ & [135] \\
\hline
\end{tabular}

\section{Discussion}

\subsection{Screening of the Studies}

The studies analyzed were restricted to genetic improvement and in line with the questions proposed in the protocol formulated for this review. For this reason, articles on FOC genetic diversity, specific management strategies, and first reports of the disease were not considered in the analyses (Figure S1). Literature reviews were also excluded from the research to avoid bias since they could overestimate data, as many articles would be repeated.

Thus, the exclusion criteria used during the extraction stage of the article screening process revealed that many specific studies on FOC genetic diversity $(n=72)$ were generated in the last ten years, as well as many articles that escaped the proposed subject of this review $(n=47)$ and several literature reviews $(n=26)$ (Figure S1). Although these papers were excluded by the criteria, they revealed important aspects of the direction of research on Fusarium wilt in the last 10 years, considering the search string used.

The considerable amount of data on the genetic diversity of FOC generated in recent years was primarily in response to the need to understand the population structure of the pathogen in different locations and the evolutionary mechanisms of the fungus that culminate in the emergence of new races $[48,136,137]$. In fact, the potential for public investment in research that addresses the dissemination of the FOC TR4 can generate high returns and substantially delay the spread of this disease [138].

\subsection{Locations of Knowledge Generation}

A substantial amount of data on banana genetic improvement for resistance to Fusarium wilt was evaluated in this systematic review, the majority (50\%) from China. This is a consequence of the number of projects to control Fusarium wilt in China, involving institutions, such as the Guangdong Academy of Agricultural Sciences (GDAAS), Chinese 
Academy of Tropical Agricultural Sciences (CATAS), Fujian Agriculture and Forestry University, Hainan Academy of Agricultural Sciences, and Guangzhou Institute of Agricultural Sciences [139]. In addition, China is among the countries in Southwest Asia where banana plants were domesticated [140], in which bananas are one of the fruits with the oldest consumption record, and the country that ranks second among the top 10 banana producers worldwide [2].

Besides China, India (16.7\%), Australia (10.4\%), and Brazil (7.3\%) have also contributed to the improvement in research on Musa spp. India is the largest banana producer globally, whereas Brazil ranks fourth among banana producers [2]. Furthermore, these countries host important research institutions, which work on banana improvement for the development of resistant hybrids from germplasm collections, such as the Brazilian collections of the National Research Center for Banana (NRCB) and EMBRAPA. Australia was the first country to report and describe Fusarium wilt and one of the first countries facing major problems with FOC TR4, which led to the end of the Cavendish banana industry in the Northern Territory in 2015 [35,141,142].

Overall, in recent years, a major stimulus for the growth in studies on Fusarium wilt has been the emergence of FOC TR4 as the most devastating threat to bananas worldwide. A clear demonstration of this is the estimate that $17 \%$ of the current banana cultivation area, with an annual production of 36 million tons worth approximately US $\$ 10$ billion at current prices, could be lost over the next 20 years because of Fusarium wilt, which would necessitate investment in research aimed at improving the crop in this scenario [138,143].

\subsection{Gene Expression Analysis}

The genome of the diploid species Musa acuminata ssp. malaccensis, which is the ancestor of most banana triploid cultivars, has been sequenced [144]. In the present study, $50 \%$ of the articles used the banana genome as a tool for analysis. For this reason, the highest frequency of articles was related to in silico studies (45\%), as part of gene expression analyses (30\%) that mostly performed RT-qPCR and PCR analyses (34\%), as well as bioinformatic analyses $(23 \%)$.

Identifying genes related to host defense is one of the first steps to understand the underlying mechanism of resistance to diseases in plants [145]. Concerning FOC, knowledge of global gene expression patterns, influenced by infection of different races, has enhanced our understanding of host responses to infection. Moreover, the availability of banana transcriptomes was highly useful to improve the annotation of the banana genome and for biological research [37]. Based on the knowledge of the global patterns of gene expression influenced by infection of FOC R1 and FOC TR4, Li et al. [37] found a large number of simple nucleotide polymorphisms (SNPs) and short insertions and deletions (indels), which previously had not been annotated in the Musa genome. Other transcriptomic studies observed the regulated expression of defense genes, cell wall-modifying genes, and a phytoalexin, flavonoids, lignin biosynthesis genes and jasmonic acid and other plant hormones and transcription factors $[37,113-117,146,147]$.

The lack of standardization pertaining to the inoculation methods for evaluating gene expression should be questioned to develop a universal method for plant host inoculation so that the results could be equated and compared. It should be noted that a striking difference between these methods is the generation of wounds in the roots before exposing them to a suspension with the fungus, which in fact does not reflect a similar situation in the field, except when there are interactions with other microorganisms in the soil, such as nematodes [107]. Therefore, we consider that the inoculation methods adopted in most studies should be reconsidered because they are primarily related to the mechanical opening of wounds made with sterile needles or crushing the roots. In addition, differences related to the concentration of spores in the infection process generated marked changes in the plant response to infection and in gene expression, especially when a high inoculation pressure is considered, such as at the concentration of $5 \times 10^{6}$ spores $\mathrm{mL}^{-1}$ associated with wound generation. Consistent with this information, we know that F. oxysporum is 
considered a hemibiotrophic pathogen because it begins its infection cycle as a biotroph and later changes to a necrotroph and as the gene expression changes that occur in the roots. Host responses may be prioritized to the perception of the pathogen, preventing the penetration of the root tissue during the biotrophic stage, which would not be possible to notice in previously injured tissue $[46,148]$. Therefore, we suggest that a standardized method should be adopted regarding the inoculation method of host plants to verify gene expression, aimed mainly to simulate situations in the system of banana cultivation, considering the mechanisms of dissemination of FOC that usually occur by movement and deposition of contaminated soil [30].

\subsection{Studies on Resistance Sources}

Although available edible banana cultivars originate from M. acuminata (genome A) and Musa balbisiana (genome B), genome B has been associated with the best vigor and tolerance to biotic and abiotic stresses and is, therefore, a target for Musa spp. improvement programs $[149,150]$. The AAA triploid genomes frequently occurred when considering all resistance sources related to both FOC R1 and FOC TR4 (Figure 8). In the studies with FOC TR4, the highest frequency of resistant genotypes were related to AA diploid genomes (Figure 9). This demonstrates that FOC TR4-related resistance sources are still mostly composed of wild diploids that have not yet been exploited for triploid cultivars, as in FOC R1, which already has a large panel of resistant cultivars available.

Thus, we have shown that some wild relatives of edible bananas, such as M. itinerans, Pahang, Calcutta 4, DH Pahang and Tuu Gia (Figure 9A), are valuable resources of resistance genes to FOC TR4 [77]. These data continue to be reaffirmed based on recent RNA-seq analyses that revealed aspects of the key responses of the relative resistance of wild banana to FOC TR4, where it could be seen that many differentially expressed genes were found in the resistant wild relative Musa acuminata ssp. Burmanicoides compared to the susceptible cultivar "Brazilian (AAA)" [147].

An example of banana resistant to FOC R1 and TR4 are the triploid banana referred to as East African Highland bananas (EAHB), which a recent study has revealed that Mchare and Matooke hybrids resistant to FOC R1 can replace susceptible cultivars in areas of production severely affected by the fungus and are important resources for the generation of resistant banana [112].

The genetic basis of resistance to FOC R1 in banana has been studied in three articles, of which Arinaitwe et al. [31] and Ahmad et al. [108] suggested that resistance to Fusarium wilt in Musa spp. is conditioned by a single dominant locus of resistance, contradicting Ssali et al. [93], who concluded that the gene was recessively inherited. However, the conclusions by Ahmad et al. [108] were based on genetic analyses that included mapping studies and not just segregation data based on phenotypic characters.

\subsection{Main Methods and Tools Adopted}

One of the most-used tools (12\%), together with the symptomatological assessments to understand Musa $\times$ FOC interaction processes, is the genetic transformation of different FOC isolates with the GFP gene. This method allows researchers to follow the movements of the fungus within the tissues and compare the colonization path used by different FOC races $[37,46,72,137,151]$. A FOC STR4 strain, transformed with the GFP gene, was used to monitor the movement of the pathogen in two susceptible cultivars, Cavendish Williams (Musa AAA) and Lady Finger (Musa AAB) [46]. Those authors detected the presence of FOC on the roots, rhizome, and outer leaf sheaths of the pseudostem before the appearance of external symptoms. Another study using this method verified that, in some cases, the banana rhizome plays an important role as a barrier to the pathogen, preventing its migration to the rest of the plant [103].

The studies carried out in greenhouses corresponded to $13 \%$ of the articles, those in greenhouses and in the field to 5\%, and those only in the field to 3\%. The articles focused only on assessing Fusarium wilt symptoms are few since, overall, this type of 
evaluation is complementary to several other analyses as a safe phenotypic confirmation of resistance. Most of the evaluation methods cited are related to the quantification of the severity of Fusarium wilt by visual categorization of the cross-sections based on the level of discoloration of the vascular tissue of the rhizome and the pseudostem of the root tissue, according to the scales mentioned in Table $2[65-67,71,77,94]$.

The greatest difference found between the rating scales adopted for analysis and confirmation of banana resistance to FOC is related to the scoring grades for the disease's severity. A universal scoring scale should be adopted, especially for comparison purposes between studies from different banana research centers, to avoid discrepant results, for example, when evaluating hybrids resulting from crossbreeding, plants obtained by transgenesis, resistance-induction, or other methods.

Although there are few studies with somaclonal variation (1\%), this is a tool that presents promising results. The Cavendish somaclone GCTCV-218 for commercial cultivation under the name of Formosona, generated in 2004 by the Taiwan Banana Research Institute, is known to be tolerant to FOC TR4 and two other somaclonal variants of Cavendish called GCTCV-53 and GCTCV-119 [152]. In a recent study, tests with these Cavendish banana somaclones in northern Mozambique revealed that GCTCV-119 was more resistant to FOC TR4, but GCTCV-218 produced better bunches [5]. Another recent study obtained, through different combinations of plant regulators in a culture medium, two somaclones of the cultivar Prata-Anã, namely T2-1 and T2-2, which presented resistance to FOC race 1 in a greenhouse, characterizing an important result for the banana cultivation in Brazil since the pathogen FOC R1is present in most banana plantations and this cultivar is preferred by Brazilian consumers [153].

In the articles analyzed, transgenesis was the most-used method (14\%), followed by resistance induction $(10 \%)$, hybridization $(4 \%)$, in vitro mutagenesis $(4 \%)$, and somaclonal variation, clone selection, and somatic embryogenesis (1\%). Although the transgenic method has a limitation related to the production of embryogenic cell suspensions, a timeconsuming process, some protocols have facilitated their implementation $[127,154]$. Among the cited protocols, the most-used have been proposed by Ganapathi et al. [154], which included the establishment of embryogenic cell cultures from thin sections of the shoot tip of cultivated Rasthali (AAB) banana cultivar in vitro and by Khanna et al. [155], which proposed transformation mediated by Agrobacterium tumefaciens assisted by centrifugation (CAAT) from male flower embryogenic cells suspensions of the Cavendish (AAA) and Lady Finger (AAB) cultivars. A protocol established by Yip et al. [69] proposes the substitution of embryogenic cell suspensions for meristematic tissue, where they use multiple shot clump (MSC) of Pei Chiao (AAA) and Gros Michel (AAA) bananas induced from shoots in the rhizome in MS medium; this could be another feasible option for banana cultivars where suspension cultures are difficult to establish. Another protocol was proposed by Subramaniam et al. [122] using the biobalistic gun method for the transformation of the 'Rastali' (AAB) banana cultivar. In addition, the availability of banana genes (cisgenes) and genes from other appropriate sources (transgenes) allowed the development and evaluation of transgenic plants (Table 6).

Conventional resistance improvement methods using hybridization between fertile diploids and crossbreeding with triploid or tetraploid cultivars are efficient. However, they have some limitations concerning the polyploid nature of the cultivars and the low female fertility, as well as the long life cycle leading to a long reproductive cycle $[156,157]$. Other challenges are related to the need for a large space, which results in high costs and limited knowledge about resistance genetics [31,158,159].

Transgenic methods permit the addition of a single gene or several genes to a highly desirable cultivar quickly [81,124-126]. Due to the sterility of these cultivars, the flow of transgenes and the crossing of modified genes between wild Musa species are unlikely; therefore, genetically modified (GM) bananas could be compatible with organic agriculture [159]. In addition, although no genome editing data associated with obtaining Fusarium wilt-resistant cultivars were identified in this study, the potential for using the 
CRISPR/Cas9, a genome-editing tool for the development of disease-resistant banana varieties, also has been reported. The use of genome editing (GE) with the availability of a whole-genome sequence and its potential applications to develop disease-resistant bananas opens new areas of research [160-165]. Although there are no published data in banana breeding, another potential tool to be applied is resistance gene enrichment sequencing (RenSeq), a technology that enables the discovery and annotation of pathogen resistance gene families in plant genome sequences. The use of this high-throughput technique was well demonstrated in wheat (Triticum estivum) [164] and potato (Solanum tuberosum) [165].

These data encourage discussions on the current status of biosafety regulations and laws on the marketing of GM products that face some challenges because of the regulation of these products in several countries [162,163]. Furthermore, their outlook indicated that investments in GM banana plantations would bring few beneficiaries, given the assumption that countries with export-oriented banana production would not adopt GM varieties because of political and consumer concerns [138]. In this sense, it seems reasonable to invest more in improvements based on crossbreeding, considering that there are sources of resistance to Fusarium wilt caused by FOC R1 and FOC TR4, which enables the selection of resistant hybrids within the progeny generated.

Using an ex-ante quantitative risk index model, Staver et al. [138] showed that investments in different research areas assessed to address the threat and projected losses from FOC TR4 would provide positive returns and contribute to a reduction in poverty. Moreover, there would be superior returns in poverty reduction, especially in Africa, in the face of investments in the research areas related to the conventional improvement of cultivars resistant to Fusarium wilt, as well as in the research area related to improving exclusion and surveillance, as well as measures to eradicate or contain the disease, with 850,000 and 807,000 people lifted out of poverty in each case, respectively.

\subsection{Perspectives}

In this study, we found that several articles in the last 10 years have focused on a variety of analyses to improve our understanding and identification of genetic, molecular, biochemical, or structural mechanisms of banana resistance to FOC, based on a set of tools. Based on these articles, we also showed that there are sources of resistance to FOC R1 and FOC TR4 in banana germplasms and that the data generated in these studies are the basis for obtaining cultivars resistant to Fusarium wilt. Moreover, they can contribute significantly to the expansion of resistant cultivars, including those for export. Although there is not yet a banana cultivar resistant to FOC TR4 that can replace the cultivars of the Cavendish subgroup, from the resistance sources found in different studies, it would be possible to develop a "type" similar to the Cavendish cultivars resistant to FOC TR4 or other races.

Concerning the improvement methods, there is a growing incentive for new precise and efficient genetic technologies, and the use of the CRISPR/Cas9 genome editing tool will also contribute to obtaining banana cultivars with FOC resistance in a short span of time. Other tools, which explore acquired and induced systemic resistance, also emerged as important means to achieve resistance to the pathogen, supported by experiments on tissue culture. Meanwhile, conventional improvement seeks to overcome the challenges inherent to the plant species by offering seemingly more appropriate measures with a focus on family-based agriculture of banana production systems worldwide. Nevertheless, the debate concerning various improvement methods should not be focused on just one method since all of them contribute to improving the crop, and the existence of different scenarios of banana production should be considered for the use of each method.

Furthermore, it is important to emphasize that the results obtained in this study are linked to the keywords used in the search string. The use of different terms could lead to the inclusion and exclusion of other articles in the systematic review and, consequently, lead to other methods and conclusions. 


\section{Conclusions}

Improvement programs of Musa spp. have sought to reinforce their methods through new technologies and accumulate knowledge on resistance to Fusarium wilt. The genome sequencing of Musa is a widely used data source for improving the identification and analysis of resistance-related genes. The production of transgenic bananas has been explored, leading to the need for social exposure regarding the acceptance of such products. Although the use of genome editing tools, such as CRISPR/Cas9, to obtain resistance to Fusarium wilt in banana plants has not been performed, it is a method with promising prospects. In this review, we highlighted sources of resistance to FOC (R1 and TR4) based on diploids resistant to Fusarium wilt, which is the starting point for genetic improvement.

Therefore, we confirm that genetic improvement is the best strategy for combating Fusarium wilt by expanding resistant cultivars to producers. From the data collected in our systematic review, we believe that future research efforts can be based on integrating the knowledge obtained thus far to obtain results with greater applicability and direct the next steps in research to produce banana species resistant to Fusarium wilt. We suggest that future studies address the following questions: How can we exploit germplasm sources resistant to FOC R1 and FOC TR4 in improvement programs? Could the standardization of protocols for plant inoculation facilitate the comparison of data regarding gene expression analysis? Should a universal scoring scale contemplating the disease's external and internal symptoms be elaborated based on existing scales? Can existing molecular markers be used in a standard-assisted selection protocol for resistance to FOC R1 and FOC TR4?

In addition, strategies based on the integration of knowledge from different Musa spp. improvement research centers should be adopted for cooperative efforts so that different improvement programs can cooperate on a global scale. Considering that the current banana export scenario is based exclusively on a single group, strategies should be considered to ensure the agribusiness export's sustainability, prioritizing the production of other cultivars resistant to FOC.

Supplementary Materials: The following are available online at https://www.mdpi.com/article/ 10.3390/jof7040249/s1, Figure S1. Frequency of articles by exclusion (E) and inclusion (I) criteria, used in the study extraction phase to composing a systematic review of the resistance of Musa spp. to Fusarium wilt; Table S1. Summarization of the main genes identified in the analysis of articles on the improvement of banana to Fusarium wilt carried out in the last ten years (additional to Figure 9B); Table S2. Origin and database of articles selected to compose a systematic review on the improvement of banana to Fusarium wilt.

Author Contributions: Conceptualization, A.d.J.R., F.H., E.P.A.; methodology, A.d.J.R.; J.M.d.S.S. and F.d.S.N.; software, A.d.J.R.; A.S.S.; validation, A.d.J.R., J.M.d.S.S., F.d.S.N., A.S.S., V.B.d.O.A., J.A.d.S.S., C.F.F., F.H. and E.P.A.; formal analysis, A.d.J.R.; investigation, A.d.J.R., J.A.d.S.-S., F.H., E.P.A.; resources, E.P.A.; data curation, A.d.J.R., J.M.d.S.S. and F.d.S.N.; writing-original draft preparation, A.d.J.R., E.P.A.; writing—review and editing, A.d.J.R., J.A.d.S.-S., C.F.F., E.P.A.; visualization, A.d.J.R., J.M.d.S.S., F.d.S.N., A.S.S., V.B.d.O.A., J.A.d.S.-S., C.F.F., F.H. and E.P.A.; supervision, E.P.A.; project administration, E.P.A.; funding acquisition, E.P.A. All authors have read and agreed to the published version of the manuscript.

Funding: This research was funded by Embrapa, grant number 22.15.11.004.00.00 (Banana Breeding Project, Phase II) and Corporación Bananera Nacional (CORBANA) grant number 5153/Funarbe (Strategic alliance to develop Cavendish-type cultivars resistant to FOC TR4).

Acknowledgments: The authors thank the Graduate Program in Biotechnology (PPGBiotec) of the State University of Feira de Santana and CNPq for the research productivity grants to Amorim EP and Ferreira CF; Fapesb for providing Ph.D. scholarships to Rocha AJ, Soares JMS, and Nascimento FS; Professor Eduardo Mizubuti (Federal University of Viçosa) for the corrections and meaningful suggestions to improve this review; and the Corporación Bananera Nacional (Corbana) for financing the publication of this review and the strategic alliance with EMBRAPA to develop Cavendish-type cultivars resistant to FOC TR4, which began in 2020. We would like to acknowledge the financial 
contribution of the CGIAR Research Program on Roots, Tubers and Bananas and the CGIAR Fund Donors for covering the open access fee of this publication.

Conflicts of Interest: The authors declare no conflict of interest.

\section{References}

1. FAOSTAT. Available online: http:/ / www.fao.org/faostat/en/\#data/QC (accessed on 4 June 2020).

2. FAO. Available online: http://www.fao.org/fileadmin/templates/est/COMM_MARKETS_MONITORING/Bananas/ Documents/Banana_Market_Review_Prelim_Results_2018.pdf. (accessed on 4 June 2020).

3. Adeniji, T.; Tenkouano, A.; Ezurike, J.; Ariyo, C.; Vroh-Bi, I. Value-adding post harvest processing of cooking bananas (Musa spp. AAB and ABB genome groups). Afri. J. Biotech. 2010, 9, 9135-9141.

4. Dotto, J.; Matemu, A.O.; Ndakidemi, P.A. Potential of cooking bananas in addressing food security in East Africa. Int. J. Biol. Sci. 2018, 13, 278-294.

5. Viljoen, A.; Mostert, D.; Chiconela, T.; Beukes, I.; Fraser, C.; Dwyer, J.; Amugoli, O.M. Occurrence and spread of the banana fungus Fusarium oxysporum f. sp. cubense TR4 in Mozambique. S. Afr. J. Sci. 2020, 116, 11-12. [CrossRef]

6. Dita, M.A.; Garming, H.; Van den Bergh, I.; Staver, C.; Lescot, T. Banana in Latin America and the Caribbean: Current state, challenges and perspectives. Acta Hortic. 2011, 986, 365-380. [CrossRef]

7. Lescot, T. Close-up Banana: Statistics. FruiTrop 2011, 189, 59-62.

8. Gomes, E.W.F.; Willadino, L.; Martins, L.S.S.; Camara, T.R. The effects of salinity on five banana genotypes (Musa spp). In Plant Nutrition; Horst, W.J., Schenk, M.K., Eds.; Springer: Dordrecht, The Netherlands, 2001; Volume 92, pp. 410-411. [CrossRef]

9. Willadino, L.; Camara, T.R.; Ribeiro, M.B.; Amaral, D.O.J.; Suassuna, F.; Silva, M.V.D. Mechanisms of tolerance to salinity in banana: Physiological, biochemical, and molecular aspects. Rev. Bras. de Frutic. 2017, 39, 1-8. [CrossRef]

10. Said, E.M.; Mahmoud, R.A.; Al-Akshar, R.; Safwat, G. Drought stress tolerance and enhancement of banana plantlets in vitro. Austin J. Biotechnol. Bioeng. 2015, 2, 1040.

11. Marssaro, A.L.; Morais-Lino, L.S.; Cruz, J.L.; Ledo, C.A.S.; Santos-Serejo, J.A. Simulation of in vitro water deficit for selecting drought-tolerant banana genotypes. Pesqui. Agropecu. Bras. 2017, 52, 1301-1304. [CrossRef]

12. Nansamba, M.; Sibiya, J.; Tumuhimbise, R.; Karamura, D.; Kubiriba, J.; Karamura, E. Karamura, E. Breeding banana (Musa spp.) for drought tolerance: A review. Plant Breed. 2020, 139, 685-696. [CrossRef]

13. Vu, T.; Sikora, R.; Hauschild, R. Fusarium oxysporum endophytes induced systemic resistance against Radopholus similis on banana. Nematology 2006, 8, 847-852. [CrossRef]

14. Gaidashova, S.V.; Uwimpuhwe, B.; Karamura, E.B. Identificatio of banana varieties with resistance to nematodes in Rwanda. Afr. Crop Sci. J. 2008, 16, 27-33. [CrossRef]

15. Tripathi, L.; Atkinson, H.; Roderick, H.; Kubiriba, J.; Tripathi, J.N. Genetically engineered bananas resistant to Xanthomonas wilt disease and nematodes. Food Energy Secur. 2017, 6, 37-47. [CrossRef]

16. Njau, N.; Mwangi, M.; Gathu, R.; Mbaka, J.; Muasya, R. Banana weevil (Cosmopolites sordidus) reduces availability of corms for seedling production through macropropagation technology. J. Anim. Plant Sci. 2011, 12, 1537-1542.

17. Arinaitwe, I.K.; Hilman, E.; Ssali, R.; Barekye, A.; Kubiriba, J.; Kagezi, G.; Talwana, H.; Nankinga, C.; Ragama, P.E.; Tushemereirwe, W.K.; et al. Response of banana hybrids to the banana weevil (Cosmopolites sordidus Germar)(Coleoptera: Curculionidae) in Uganda. Uganda J. Agric. Sci. 2014, 15, 73-85.

18. Twesigye, C.K.; Ssekatawa, K.; Kiggundu, A.; Tushemereirwe, W.; Matovu, E.; Karamura, E. Corm damage caused by banana weevils Cosmopolites sordidus (Germar) collected from different banana growing regions in Uganda. Agric. Food Secur. 2018, 7 , 1-8. [CrossRef]

19. Monteiro, J.D.; Santos, M.; Santos, J.R.P.; Cares, J.E.; Marchão, R.L.; Amorim, E.P.; Costa, D.D.C. Identification of plant parasitic nematodes in triploid and tetraploid bananas in brazil. Rev. Caatinga 2020, 33, 865-877. [CrossRef]

20. Galvez, L.C.; Barbosa, C.F.C.; Koh, R.B.L.; Aquino, V.M. Loop-mediated isothermal amplification (LAMP) assays for the detection of abaca bunchy top virus and banana bunchy top virus in abaca. Crop. Prot. 2020, 131, 105101. [CrossRef]

21. Sairam, S.; Selvarajan, R.; Handanahalli, S.S.; Venkataraman, S.; Sairam, S.; Selvarajan, R.; Handanahalli, S.S.; Venkataraman, S. Towards understanding the structure of the capsid of Banana Bunchy Top Virus. BioRxiv 2020. [CrossRef]

22. Tripathi, L.; Tripathi, J.N.; Tushemereirwe, W.K. Strategies for resistance to bacterial wilt disease of bananas through genetic engineering. Afr. J. Biotechnol. 2004, 3, 688-692.

23. Nakato, V.; Mahuku, G.; Coutinho, T. Xanthomonas campestris pv. musacearum: A major constraint to banana, plantain and enset production in central and east Africa over the past decade. Mol. Plant. Pathol. 2018, 19, 525-536. [CrossRef]

24. Geberewold, A.Z. Review on impact of banana bacterial wilt (Xhantomonas campestris pv. Musacerum) in East and Central Africa. Cogent Food Agric. 2019, 5, 1586075. [CrossRef]

25. Studholme, D.J.; Wicker, E.; Abrare, S.M.; Aspin, A.; Bogdanove, A.; Broders, K.; Dubrow, Z.; Grant, M.; Jones, J.B.; Karamura, G.; et al. Transfer of Xanthomonas campestris pv. arecae and X. campestris pv. musacearum to X. vasicola (Vauterin) as X. vasicola pv. arecae comb. nov. and X. vasicola pv. musacearum comb. nov. and description of X. vasicola pv. vasculorum pv. nov. Phytopathology 2020, 110, 1153-1160. [CrossRef] [PubMed] 
26. Timm, S.E.; Pardo, H.L.; Coello, P.R.; Navarrete, C.T.; Villegas, N.O.; Ordóñez, S.E. Identification of differentially-expressed genes in response to Mycosphaerella fijiensis in the resistant Musa accession 'Calcutta-4'using suppression subtractive hybridization. PLOS ONE 2016, 11, 1-17. [CrossRef]

27. Arango Isaza, R.E.; Diaz-Trujillo, C.; Dhillon, B.; Aerts, A.; Carlier, J.; Crane, C.F.; Jong, T.V.; Vries, I.; Dietrich, R.; Farmer, A.D.; et al. Combating a global threat to a clonal crop: Banana black Sigatoka pathogen Pseudocercospora fijiensis (synonym Mycosphaerella fijiensis) genomes reveal clues for disease control. Plos Genet. 2016, 12, e1005876. [CrossRef]

28. Vázquez-Euán, R.; Chi-Manzanero, B.; Hernández-Velázquez, I.; Tzec-Simá, M.; Islas-Flores, I.; Martínez-Bolaños, L.; CantoCanché, B. Identification of new hosts of pseudocercospora fijiensis suggests innovative pest management programs for black sigatoka disease in banana plantations. Agronomy 2019, 9, 666. [CrossRef]

29. Nascimento, F.D.S.; Sousa, Y.M.; Rocha, A.D.J.; Ferreira, C.F.; Haddad, F.; Amorim, E.P. Sources of black Sigatoka resistance in wild banana diploids. Rev. Bras. Frutic 2020, 42. [CrossRef]

30. Dita, M.; Barquero, M.; Heck, D.; Mizubuti, E.S.; Staver, C.P. Fusarium wilt of banana: Current knowledge on epidemiology and research needs toward sustainable disease management. Front. Plant. Sci. 2018, 9, 1468. [CrossRef]

31. Arinaitwe, I.K.; Teo, C.H.; Kayat, F.; Tumuhimbise, R.; Uwimana, B.; Kubiriba, J.; Swennen, R.; Harikrishna, J.A.; Othman, R.Y. Evaluation of banana germplasm and genetic analysis of an F1 population for resistance to Fusarium oxysporum $\mathrm{f}$. sp. cubense race 1. Euphytica 2019,215, 175. [CrossRef]

32. Sun, J.; Zhang, J.; Fang, H.; Peng, L.; Wei, S.; Li, C.; Lu, J. Comparative transcriptome analysis reveals resistance-related genes and pathways in Musa acuminata banana 'Guijiao 9' in response to Fusarium wilt. Plant. Physiol. Biochem. 2019, 141, 83-94. [CrossRef]

33. Gonçalves, Z.S.; Haddad, F.; Amorim, V.B.O.; Ferreira, C.F.; Oliveira, S.A.S.; Amorim, E.P. Agronomic characterization and identification of banana genotypes resistant to Fusarium wilt race 1. Eur. J. Plant. Pathol. 2019, 155, 1093-1103. [CrossRef]

34. Ploetz, R.C.; Kema, G.H.; Ma, L.J. Impact of diseases on export and smallholder production of banana. Annu. Rev. Phytopathol. 2015, 53, 269-288. [CrossRef]

35. Pegg, K.G.; Coates, L.M.; O’Neill, W.T.; Turner, D.W. The epidemiology of Fusarium wilt of banana. Front. Plant. Sci. 2019, 10, 1395. [CrossRef]

36. Ploetz, R.C. Fusarium wilt. In Handbook of Diseases of Banana, Abacá and Ense; Jones, D., Ed.; CABI Publishing: Wallingford, UK, 2019; pp. 207-228.

37. Li, C.; Shao, J.; Wang, Y.; Li, W.; Guo, D.; Yan, B.; Xia, Y.; Peng, M. Analysis of banana transcriptome and global gene expression profiles in banana roots in response to infection by race 1 and tropical race 4 of Fusarium oxysporum f. sp. cubense. BMC Genom. 2013, 14, 851. [CrossRef]

38. Lopes, O.P.; Maia, V.M.; Xavier, A.A.; Costa, M.R.D.; Rodrigues, M.G.V. Diversidade genética, crescimento e produção de genótipos de bananeira 'Prata-Anã' em área com mal do panamá. Rev. Bras. Frutic. 2014, 36, 924-939. [CrossRef]

39. Stover, R.H. Fusarial Wilt (Panama Disease) of Bananas and Other Musa Species; Commonwealth Mycological Institute: Kew, UK, 1962; p. 117.

40. Ploetz, R.C. Fusarium wilt of banana is caused by several pathogens referred to as Fusarium oxysporum f. sp. cubense. Phytopathology 2006, 96, 653-656. [CrossRef]

41. Ploetz, R.; Freeman, S.; Konkol, J.; Al-Abed, A.; Naser, Z.; Shalan, K.; Israeli, Y. Tropical race 4 of Panama disease in the Middle East. Phytoparasitica 2015, 43, 283-293. [CrossRef]

42. García-Bastidas, F.A.; Quintero-Vargas, J.C.; Ayala-Vasquez, M.; Schermer, T.; Seidl, M.F.; Santos-Paiva, M.; Noguera, A.M.; Aguilera-Galvez, C.; Wittenberg, A.; Hofstede, R.; et al. First report of Fusarium wilt Tropical Race 4 in Cavendish bananas caused by Fusarium odoratissimum in Colombia. Plant. Dis. 2020, 104, 994. [CrossRef]

43. FAO. Available online: http://www.fao.org/3/ca6911en/CA6911EN_TR4EN.pdf (accessed on 4 June 2020).

44. Thangavelu, R.; Edwin Raj, E.; Loganathan, M.; Pushpakanth, P.; Uma, S. Draft genome of Fusarium oxysporum f. sp. cubense strain Tropical Race-4 infecting Cavendish (AAA) group of banana in India. Plant. Dis. 2020, 105, 481-483. [CrossRef]

45. Gang, G.; Bizun, W.; Weihong, M.; Xiaofen, L.; Xiaolin, Y.; Chaohua, Z.; Jianhong, M.; Huicai, Z. Biocontrol of Fusarium wilt of banana: Key influence factors and strategies. Afr. J. Microbiol. Res. 2013, 7, 4835-4843. [CrossRef]

46. Warman, N.M.; Aitken, E.A. The movement of Fusarium oxysporum f. sp. cubense (sub-tropical race 4) in susceptible cultivars of banana. Front. Plant. Sci 2018, 9, 1748. [CrossRef]

47. Mostert, D.; Molina, A.B.; Daniells, J.; Fourie, G.; Hermanto, C.; Chao, C.P.; Fabregar, E.; Sinohin, V.G.S.; Masdek, N.; Thangavelu, R.A.; et al. The distribution and host range of the banana Fusarium wilt fungus, Fusarium oxysporum f. sp. cubense, in Asia. PLoS ONE 2017, 12, e0181630. [CrossRef]

48. Costa, S.N.; Bragança, C.A.D.; Ribeiro, L.R.; Amorim, E.P.; Oliveira, S.A.S.; Dita, M.A.; Laranjeira, F.F.; Haddad, F. Genetic structure of Fusarium oxysporum f. sp. cubense in different regions from Brazil. Plant. Pathol. 2015, 64, 137-146. [CrossRef]

49. Fourie, G.; Steenkamp, E.T.; Ploetz, R.C.; Gordon, T.R.; Viljoen, A. Current status of the taxonomic position of Fusarium oxysporum formae specialis cubense within the Fusarium oxysporum complex. Infect. Genet. Evol. 2011, 11, 533-542. [CrossRef] [PubMed]

50. Amorim, E.P.; Amorim, V.B.O.; Silva, M.S.; Haddad, F.; Ferreira, C.F.; Santos-Serejo, J.A. Developing hybrid banana varieties with improved properties. In Achieving Sustainable Cultivation of Bananas: Germplasm and Genetic Improvement; Kema, G.H.J., Drenth, A., Eds.; Burleigh Dodds Science Publishing: Cambridge, UK, 2021; Volume 2, pp. 1-17, ISBN 9781786763440. 
51. Wang, F.; Xia, L.; Shun, L.V.; Xu, C.; Niu, Y.; Liu, W.; Bei, H.U. Development of a mitochondrial SCAR marker related to susceptibility of banana (Musa AAA Cavendish) to Fusarium oxysporum f. sp. cubense race 4. Not. Bot. Horti. Agrobot. Cluj-Napoca 2018, 46, 509-516. [CrossRef]

52. Ghag, S.B.; Shekhawat, U.K.; Ganapathi, T.R. Fusarium wilt of banana: Biology, epidemiology and management. Int. J. Pest. Manag. 2015, 61, 250-263. [CrossRef]

53. Ploetz, R.C. Management of Fusarium wilt of banana: A review with special reference to tropical race 4. J. Crop. Prot 2015, 73, 7-15. [CrossRef]

54. Thangavelu, R.; Loganathan, M.; Arthee, R.; Prabakaran, M.; Uma, S. Fusarium wilt: A threat to banana cultivation and its management. Cab Rev. 2020, 15, 1-24. [CrossRef]

55. Bubici, G.; Kaushal, M.; Prigigallo, M.I.; Cabanás, C.G.L.; Mercado-Blanco, J. Biological control agents against Fusarium wilt of banana. Front. Microbiol. 2019, 10, 616. [CrossRef]

56. Siamak, S.B.; Zheng, S. Banana Fusarium wilt (Fusarium oxysporum f. sp. cubense) control and resistance, in the context of developing wilt-resistant bananas within sustainable production systems. Hortic. Plant J. 2018, 4, 208-218. [CrossRef]

57. Sipen, P.; Chubo, J.K.; King, P.J.; Huat, O.K.; Davey, M.R. Genetic improvement of banana using conventional and in vitro technologies. J. Crop. Improv. 2011, 25, 697-727. [CrossRef]

58. Dash, P.K.; Rai, R. Translating the "Banana genome" to delineate stress resistance, dwarfing, parthenocarpy and mechanisms of fruit ripening. Front. Plant. Sci. 2016, 7, 1543. [CrossRef]

59. Newman, M.; Gough, D. Systematic Reviews in Educational Research: Methodology, Perspectives and Application. In Systematic Reviews in Educational Research; Zawacki-Richter, O., Bedenlier, S., Buntins, K., Kerres, M., Bond, M., Eds.; Springer VS: Wiesbaden, Germany, 2020; pp. 3-22.

60. Silva, F.A.F.; Brito, B.B.; Santos, M.L.C.; Marques, H.S.; Silva, R.T., Jr.; Carvalho, L.S.D.; Vieira, E.S.; Oliveira, M.V.; Melo, F.F.D. COVID-19 gastrointestinal manifestations: A systematic review. Rev. Soc. Bras. Med. Trop. 2020, 53, e20200714. [CrossRef]

61. Munn, Z.; Stern, C.; Aromataris, E.; Lockwood, C.; Jordan, Z. What kind of systematic review should I conduct? A proposed typology and guidance for systematic reviewers in the medical and health sciences. BMC Med. Res. Methodol. 2018, 18, 1-9. [CrossRef]

62. Santos, A.S.; Amorim, E.P.; Ferreira, C.F.; Pirovani, C.P. Water stress in Musa spp.: A systematic review. PLoS ONE 2018, 13, e0208052. [CrossRef]

63. Falcomer, A.L.; Riquette, R.F.R.; Lima, B.R.; Ginani, V.C.; Zandonadi, R.P. Health benefits of green banana consumption: A systematic review. Nutrients 2019, 11, 1222. [CrossRef]

64. Maryani, N.; Lombard, L.; Poerba, Y.S.; Subandiyah, S.; Crous, P.W.; Kema, G.H.J. Phylogeny and genetic diversity of the banana Fusarium wilt pathogen Fusarium oxysporum f. sp. cubense in the Indonesian centre of origin. Stud. Myco. 2019, 92, 155-194. [CrossRef]

65. Orjeda, G. Evaluation of Musa germplasm for resistance to Sigatoka diseases and Fusarium wilt. In INIBAP Technical Guidelines 3; International Plant Genetics Resources Institute: Rome, Italy; International Network for the Improvement of Banana and Plantain: Montpellier, France; ACP-EU Technical Centre for Agriculture and Rural Cooperation: Wageningen, The Netherlands, 1998 ; p. 63.

66. Carlier, J.; De Waele, D.; Escalant, J.V. Global Evaluation of Musa Germplasm for Resistance to Fusarium Wilt, Mycosphaerella Leaf Spot Diseases and Nematodes: In-Depth Evaluation; The International Network for the Improvement of Banana and Plantain: Montpellier, France, 2002; p. 64, ISBN 2910810526.

67. Cordeiro, Z.J.M.; Shepherd, K.; Soares Filho, W.S.; Dantas, J.L.L. Avaliação de resistência ao mal-do-Panamá em híbridos tetraploides de bananeira. Fitopatol. Bras. 1993, 18, 478-483.

68. Mak, C.; Mohamed, A.A.; Liew, K.W.; Ho, Y.W. Early screening technique for Fusarium wilt resistance in banana micropropagated plants. In Banana Improvement: Cellular, Molecular Biology, and Induced Mutations; Swennen, R., Jain, M.S., Eds.; Science Publishers, Inc.: Enfield, NH, USA, 2004; pp. 219-227, ISBN 1578083400.

69. Yip, M.K.; Lee, S.W.; Su, K.C.; Lin, Y.H.; Chen, T.Y.; Feng, T.Y. An easy and efficient protocol in the production of pflp transgenic banana against Fusarium wilt. Plant. Biotechnol. Rep. 2011, 5, 245-254. [CrossRef]

70. Orr, R.; Pattison, A.; East, D.; Warman, N.; O’Neill, W.; Czislowski, E.; Nelson, P.N. Image-based quantification of Fusarium wilt severity in banana. Australas. Plant. Dis. Notes 2019, 14, 14. [CrossRef]

71. Viljoen, A.; Mahuku, G.; Massawe, C.; Ssali, R.T.; Kimunye, J.; Mostert, G.; Ndayihanzamaso, P.; Coyne, D.L. Banana Pests and Diseases: Field Guide for Disease Diagnostics and Data Collection; International Institute of Tropical Agriculture (IITA): Ibadan, Nigeria, 2017; p. 73.

72. Chen, A.; Sun, J.; Matthews, A.; Armas-Egas, L.; Chen, N.; Hamill, S.; Mintoff, S.; Tran-Nguyen, L.T.T.; Batley, J.; Aitken, E.A.B. Assessing variations in host resistance to Fusarium oxysporum $\mathrm{f}$ sp. cubense race 4 in Musa species, with a focus on the subtropical race 4. Front. Microbiol. 2019, 10, 1062. [CrossRef]

73. Baharum, N.A.; Othman, R.Y.; Mohd-Yusuf, Y.; Tan, B.C.; Zaidi, K.; Khalid, N. The Effect of Pathogenesis-Related 10 (Pr-10) Gene on the Progression of Fusarium Wilt in Musa acuminata cv. Berangan. Sains Malays. 2018, 47, 2291-2300. [CrossRef]

74. Zhang, L.; Yuan, T.; Wang, Y.; Zhang, D.; Bai, T.; Xu, S.; Wang, Y.; Tang, W.; Zheng, S.J. Identification and evaluation of resistance to Fusarium oxysporum f. sp. cubense tropical race 4 in Musa acuminata Pahang. Euphytica 2018, 106, 1-12. [CrossRef]

75. Li, W.M.; Dita, M.; Wu, W.; Hu, G.B.; Xie, J.H.; Ge, X.J. Resistance sources to Fusarium oxysporum f. sp. cubense tropical race 4 in banana wild relatives. Plant. Pathol. 2015, 64, 1061-1067. [CrossRef] 
76. Sun, D.; Lu, X.; Hu, Y.; Li, W.; Hong, K.; Mo, Y.; Xie, J. Methyl jasmonate induced defense responses increase resistance to Fusarium oxysporum f. sp. cubense race 4 in banana. Sci Hortic. 2013, 164, 484-491. [CrossRef]

77. Zuo, C.; Deng, G.; Li, B.; Huo, H.; Li, C.; Hu, C.; Kuang, R.; Yang, Q.; Dong, T.; Sheng, O.; et al. Germplasm screening of Musa spp. for resistance to Fusarium oxysporum f. sp. cubense tropical race 4 (Foc TR4). Eur. J. Plant. Pathol. 2018, 151, 723-734. [CrossRef]

78. Ribeiro, L.R.; Oliveira, S.A.S.D.; Amorim, E.P.; Serejo, J.A.S.; Haddad, F. Sources of resistance to Fusarium oxysporum f. sp. cubense in banana germplasm. Rev. Bras. Frutic. 2018, 40, 1-8. [CrossRef]

79. Mohamed, A.A.; Mak, C.; Liew, K.W.; Ho, Y.W. Early evaluation of banana plants at nursery stage for Fusarium wilt tolerance. In Fusarium Wilt Management: Towards Sustainable Cultivation, Proceedings of the International Workshop Banana Fusarium Wilt Diseases, Genting Highlands Resort, Malaysia, 18-20 October 1999; Molina, A., Mak, C., Liew, K.W., Ho, Y.W., Masdek, N.K., Liew, K.W., Eds.; Bioversity International: Rome, Italy, 2001.

80. Wei, Y.; Hu, W.; Wang, Q.; Zeng, H.; Li, X.; Yan, Y.; Reiter, R.J.; He, C.; Shi, H. Identification, transcriptional and functional analysis of heat-shock protein 90s in banana (Musa acuminata L.) highlight their novel role in melatonin-mediated plant response to Fusarium wilt. J. Pineal Res. 2016, 62, e12367. [CrossRef]

81. Garcez, M.; Martins, J.A.S.; Rodrigues, E.J.R. Evaluation of different banana genotypes for resistance to panama disease. Biosci. J. 2016, 32, 431-435. [CrossRef]

82. Rodriguez, M.A.D.; Ribeiro, L.; Amorim, E.P.; Cordeiro, Z.J.M.; Silva, S.O. Metodologia Para a Caracterização de Genótipos de Bananeira Quanto à Resistência ao Mal.-do-Panamá em Casa de Vegetação; Embrapa Mandioca e Fruticultura. Comunicado Técnico, 150; Embrapa Mandioca e Fruticultura: Cruz das Almas, Brazil, 2011; 5p.

83. Ghag, S.B.; Shekhawat, U.K.; Ganapathi, T.R. Host-induced post-transcriptional hairpin RNA-mediated gene silencing of vital fungal genes confers efficient resistance against Fusarium wilt in banana. Plant. Biotechnol. J. 2014, 12, 541-553. [CrossRef]

84. Smith, M.K.; Langdon, P.W.; Pegg, K.G.; Daniells, J.W. Growth, yield and Fusarium wilt resistance of six FHIA tetraploid bananas (Musa spp.) grown in the Australian subtropics. Sci. Hortic. 2014, 170, 176-181. [CrossRef]

85. Jones, D.R. The Improvement and Testing of Musa: A Global Partnership. Inter-National Network for the Improvement of Banana and Plantain; International Network for the Improvement of Banana and Plantain (INIBAP): Montpellier, France, $1994 ;$ p. 303.

86. Mohandas, S.; Sowmya, H.D.; Saxena, A.K.; Meenakshi, S.; Rani, R.T.; Mahmood, R. Transgenic banana cv. Rasthali (AAB, Silk gp) harboring Ace-AMP1 gene imparts enhanced resistance to Fusarium oxysporum f. sp. cubense race 1. Sci. Hortic. 2013, 164, 392-399. [CrossRef]

87. Nasir, N.; Pittaway, P.A.; Pegg, K.G.; Lisle, A.T. A foliar rating system for com-paring the resistance of banana cultivar grown as tissue cultured plantlets in the laboratory to Fusarium wilt. Plant. Pathol. 2003, 32, 521-526. [CrossRef]

88. Ting, A.S.Y.; Mah, S.W.; Tee, C.S. Evaluating the feasibility of induced host resistance by endophytic isolate Penicillium citrinum BTF08 as a control mechanism for Fusarium wilt in banana plantlets. Biol. Control. 2012, 61, 155-159. [CrossRef]

89. Paul, J.Y.; Becker, D.K.; Dickman, M.B.; Harding, R.M.; Khanna, H.K.; Dale, J.L. Apoptosis-related genes confer resistance to Fusarium wilt in transgenic 'Lady Finger'bananas. Plant. Biotechnol. J. 2011, 9, 1141-1148. [CrossRef]

90. Sun, J.B.; Peng, M.; Wang, Y.G.; Zhao, P.J.; Xia, Q.Y. Isolation and characterization of antagonistic bacteria against fusarium wilt and induction of defense related enzymes in banana. Afr. J. Microbiol. Res. 2011, 5, 509-515. [CrossRef]

91. Ploetz, R.C.; Vazquez, A.; Haynes, J.L. Response of new banana accessions in South Florida to Panama disease. J. Crop. Prot. 1999, 18, 445-449. [CrossRef]

92. Wu, Y.L.; Yi, G.J.; Peng, X.X. Rapid screening of Musa species for resistance to Fusarium wilt in an in vitro bioassay. Eur. J. Plant. Pathol. 2010, 128, 409-415. [CrossRef]

93. Ssali, R.T.; Kiggundu, A.; Lorenzen, J.; Karamura, E.; Tushemereirwe, W.; Viljoen, A. Inheritance of resistance to Fusarium oxysporum f. sp. cubense race 1 in bananas. Euphytica 2013, 194, 425-430. [CrossRef]

94. Smith, L.J.; Smith, M.K.; Tree, D.; O’Keefe, D.; Galea, V.J. Development of a small-plant bioassay to assess banana grown from tissue culture for consistent infection by Fusarium oxysporum f. sp. cubense. Australas. Plant. Path. 2008, 37, 171-179. [CrossRef]

95. Li, W.; Ge, X.; Wu, W.; Wang, W.; Hu, Y.; Mo, Y.; Sun, D.; Shi, S.; Xie, J. Identification of defense-related genes in banana roots infected by Fusarium oxysporum f. sp. cubense tropical race 4. Euphytica 2015, 205, 837-849. [CrossRef]

96. Ghag, S.B.; Shekhawat, U.K.S.; Ganapathi, T.R. Native cell-death genes as candidates for developing wilt resistance in transgenic banana plants. Aob Plants 2014, 6, plu037. [CrossRef]

97. Saraswathi, M.S.; Kannan, G.; Uma, S.; Thangavelu, R.; Backiyarani, S. Improvement of banana cv. Rasthali (Silk, AAB) against Fusarium oxysporum f. sp. cubense (VCG 0124/5) through induced mutagenesis: Determination of LD 50 specific to mutagen, explants, toxins and in vitro and in vivo screening for Fusarium wilt resistance. Indian J. Exp. Biol. 2016, 54, 345-353. [PubMed]

98. Ghag, S.B.; Shekhawat, U.K.; Ganapathi, T.R. Characterization of Fusarium wilt resistant somaclonal variants of banana cv. Rasthali by cDNA-RAPD. Mol. Biol. Rep. 2014, 41, 7929-7935. [CrossRef] [PubMed]

99. Wu, Y.; Yi, G.; Peng, X.; Huang, B.; Liu, E.; Zhang, J. Systemic acquired resistance in Cavendish banana induced by infection with an incompatible strain of Fusarium oxysporum f. sp. cubense. J. Plant. Physiol. 2013, 170, 1039-1046. [CrossRef]

100. Magambo, B.; Harjeet, K.; Arinaitwe, G.; Tendo, S.; Arinaitwe, I.K.; Kubiriba, J.; Tushemereirwe, W.; Dale, J. Inhibition of cell death as an approach for development of transgenic resistance against Fusarium wilt disease. Afr. J. Biotechnol. 2016, 15, 786-797. [CrossRef] 
101. Smith, M.K.; Daniells, J.W.; Peasley, D.; O’Neill, W.; Samuelian, S.; Wright, C.; Drenth, A. Field evaluation of six Gros Michel banana accessions (Musa spp., AAA group) for agronomic performance, resistance to Fusarium wilt race 1 and yellow Sigatoka. J. Crop. Prot. 2018, 113, 84-89. [CrossRef]

102. Garcia-Bastidas, F.A.; Van der Veen, A.; Nakasato-Tagami, G.; Meijer, H.J.; Arango-Isaza, R.E.; Kema, G.H. An improved phenotyping protocol for panama disease in banana. Front. Plant. Sci. 2019, 10, 1006. [CrossRef]

103. Cheng, C.; Liu, F.; Sun, X.; Tian, N.; Mensah, R.A.; Li, D.; Lai, Z. Identification of Fusarium oxysporum f. sp. cubense tropical race 4 (Foc TR4) responsive miRNAs in banana root. Sci. Rep. 2019, 9, 1-16. [CrossRef]

104. Dita, M.A.; Pérez Vicente, L.; Martínez, E. Inoculation of Fusarium oxysporum f. sp. cubense causal agent of fusarium wilt in banana. In Technical Manual: Prevention and Diagnostic of Fusarium Wilt of Banana Caused by Fusarium Oxysporum f. sp. cubense Tropical Race 4 (TR4); Pérez Vicente, L., Dita, M.A., de la Parte, E.M., Eds.; Food and Agriculture Organization of the United Nations: Rome, Italy, 2014; pp. 55-58.

105. Buregyeya, H.; Tumuhimbise, R.; Matovu, M.; Tumwesigye, K.S.; Kubiriba, J.; Nowankunda, K.; Tushemereirwe, W.K.; Karamura, D.; Karamura, E.; Kityo, R.M.; et al. Fusarium oxysporum Race 1 resistance and quality traits variations in apple banana germplasm. J. Plant. Breed. Crop. Sci. 2020, 12, 16-24. [CrossRef]

106. Sunisha, C.; Sowmya, H.D.; Usharani, T.R.; Umesha, M.; Gopalkrishna, H.R.; Saxena, A. Deployment of Stacked Antimicrobial Genes in Banana for Stable Tolerance Against Fusarium oxysporum f. sp. cubense Through Genetic Transformation. Mol. Biotechnol. 2020, 62, 8-17. [CrossRef]

107. Rocha, A.D.J.; Ferreira, M.D.S.; Rocha, L.D.S.; Oliveira, A.S.; Amorim, E.P.; Mizubuti, E.S.; Haddad, F. Interaction between Fusarium oxysporum f. sp. cubense and Radopholus similis can lead to changes in the resistance of banana cultivars to Fusarium wilt. Eur. J. Plant. Pathol. 2020, 158, 403-417. [CrossRef]

108. Ahmad, F.; Martawi, N.M.; Poerba, Y.S.; Jong, H.; Schouten, H.; Kema, G.H. Genetic mapping of Fusarium wilt resistance in a wild banana Musa acuminata ssp. malaccensis accession. Appl. Genet. 2020, 133, 3409-3418. [CrossRef]

109. Silva, P.R.O.; de Jesus, O.N.; Bragança, C.A.D.; Haddad, F.; Amorim, E.P.; Ferreira, C.F. Development of a thematic collection of Musa spp accessions using SCAR markers for preventive breeding against Fusarium oxysporum f. sp cubense tropical race 4 . Genet. Mol. Res. 2016, 15, 15017765. [CrossRef]

110. Wang, W.; Hu, Y.; Sun, D.; Staehelin, C.; Xin, D.; Xie, J. Identification and evaluation of two diagnostic markers linked to Fusarium wilt resistance (race 4) in banana (Musa spp.). Mol. Bio. Rep. 2012, 39, 451-459. [CrossRef]

111. Cunha, C.M.; Hinz, R.H.; Pereira, A.; Tcacenco, F.A.; Paulino, E.C.; Stadnik, M.J.A. SCAR Marker for identifying susceptibility to Fusarium oxysporum f. sp. cubense in banana. Sci. Hortic. 2015, 191, 108-112. [CrossRef]

112. Ndayihanzamaso, P.; Mostert, D.; Matthews, M.C.; Mahuku, G.; Jomanga, K.; Mpanda, H.J.; Mduma, H.; Brown, A.; Uwimana, B.; Swennen, R.; et al. Evaluation of Mchare and Matooke Bananas for Resistance to Fusarium oxysporum f. sp. cubense Race 1. Plants 2020, 9, 1082. [CrossRef]

113. Bai, T.T.; Xie, W.B.; Zhou, P.P.; Wu, Z.L.; Xiao, W.C.; Zhou, L.; Sun, L.; Ruan, X.L.; Li, H.P.; Tumuhimbise, R.; et al. Transcriptome and expression profile analysis of highly resistant and susceptible banana roots challenged with Fusarium oxysporum $\mathrm{f}$. sp. cubense tropical race 4. PLoS ONE 2013, 8, e73945. [CrossRef]

114. Zhang, L.; Cenci, A.; Rouard, M.; Zhang, D.; Wang, Y.; Tang, W.; Zheng, S.J. Transcriptomic analysis of resistant and susceptible banana corms in response to infection by Fusarium oxysporum f. sp. cubense tropical race 4. Sci. Rep. 2019, 9, 1-14. [CrossRef]

115. Wang, Y.; Xia, Q.; Wang, G.; Zhang, H.; Lu, X.; Sun, J.; Zhang, X. Differential gene expression in banana roots in response to Fusarium wilt. Can. J. Plant. Pathol. 2017, 39, 163-175. [CrossRef]

116. Wang, Z.; Zhang, J.; Jia, C.; Liu, J.; Li, Y.; Yin, X.; Xu, B.; Jin, Z. De novo characterization of the banana root transcriptome and analysis of gene expression under Fusarium oxysporum f. sp. Cubense tropical race 4 infection. BMC Genom. 2012, 13, 650. [CrossRef]

117. Li, C.Y.; Deng, G.M.; Yang, J.; Viljoen, A.; Jin, Y.; Kuang, R.B.; Zuo, C.W.; Lv, Z.C.; Yang, Q.S.; Sheng, O.; et al. Transcriptome profiling of resistant and susceptible Cavendish banana roots following inoculation with Fusarium oxysporum $\mathrm{f}$. sp. cubense tropical race 4. BMC Genom. 2012, 13, 374. [CrossRef]

118. Fei, S.; Czislowski, E.; Fletcher, S.; Peters, J.; Batley, J.; Aitken, E.; Mitter, N. Small RNA profiling of Cavendish banana roots inoculated with Fusarium oxysporum f. sp. cubense race 1 and tropical race 4. Phytopathol. Res. 2019, 1, 22. [CrossRef]

119. Song, S.; Chen, X.; Huang, D.; Xu, Y.; Zeng, H.; Hu, X.; Wang, W. Identification of miRNAs differentially expressed in Fusarium wilt-resistant and susceptible banana varieties. S. Afr. J. Bot. 2016, 106, 244-249. [CrossRef]

120. Li, W.; Li, C.; Li, S.; Peng, M. Long noncoding RNAs that respond to Fusarium oxysporum infection in 'Cavendish' banana (Musa acuminata). Sci. Rep. 2017, 7, 1-13. [CrossRef]

121. Niu, Y.; Hu, B.; Li, X.; Chen, H.; Takáč, T.; Šamaj, J.; Xu, C. Comparative digital gene expression analysis of tissue-cultured plantlets of highly resistant and susceptible banana cultivars in response to Fusarium oxysporum. Int. J. Mol. Sci. 2018, 19, 350. [CrossRef]

122. Subramaniam, S.; Mahmood, M.; Meon, S.; Rathinam, X. Genetic engineering for tolerance to Fusarium wilt race 1 in Musa sapientum cv. Rastali (AAB) using biolistic gun transformation system. Tree For. Sci. Biotechnol. 2010, 4, 65-75.

123. Poon, N.K.; Teo, C.H. Fusarium Wilt Disease of Banana: Current Development of Fusarium Resistant Banana. J. Microbiol. Biotechnol. 2019, 4, 000134. [CrossRef] 
124. Ghag, S.B.; Shekhawat, U.K.S.; Ganapathi, T.R. Petunia floral defensins with unique prodomains as novel candidates for development of Fusarium wilt resistance in transgenic banana plants. PLoS ONE 2012, 7, e39557. [CrossRef]

125. Hu, C.H.; Wei, Y.R.; Huang, Y.H.; Yi, G.J. An efficient protocol for the production of chit42 transgenic Furenzhi banana (Musa spp. AA group) resistant to Fusarium oxysporum. In Vitro Cell. Dev. Biol. Plant 2013, 49, 584-592. [CrossRef]

126. Ghag, S.B.; Shekhawat, U.K.S.; Ganapathi, T.R. Transgenic banana plants expressing a Stellaria media defensin gene (Sm-AMP-D1) demonstrate improved resistance to Fusarium oxysporum. Plant. Cell Tissue Organ. Cult. 2014, 119, 247-255. [CrossRef]

127. Dale, J.; James, A.; Paul, J.Y.; Khanna, H.; Smith, M.; Peraza-Echeverria, S.; Garcia-Bastidas, F.; Kema, G.; Waterhouse, P.; Mengersen, K.; et al. Transgenic Cavendish bananas with resistance to Fusarium wilt tropical race 4. Nat. Commun. 2017, 8, 1-8. [CrossRef]

128. Dou, T.; Shao, X.; Hu, C.; Liu, S.; Sheng, O.; Bi, F.; Deng, G.; Ding, L.; Li, C.; Dong, T.; et al. Host-induced gene silencing of Foc TR 4 ERG 6/11 genes exhibits superior resistance to Fusarium wilt of banana. Plant. Biotechnol. J. 2020, 18, 11-13. [CrossRef]

129. Ghag, S.B.; Shekhawat, U.K.; Hadapad, A.B.; Ganapathi, T.R. Stacking of host-induced gene silencing mediated resistance to banana bunchy top virus and fusarium wilt disease in transgenic banana plants. Curr. Trends Biotechnol. Pharm. 2015, 9, 212-221.

130. Zhang, L.; Liu, L.; Cheng, P.; Shen, H.; Rong, B.; Liu, W.; Yu, G. Identification and validation of reference genes for RT-qPCR analysis in banana (Musa spp.) under Fusarium wilt resistance induction conditions. J. Phytopathol. 2017, 165, 746-754. [CrossRef]

131. Chandrasekaran, M.; Raman, C.; Karthikeyan, K.; Paramasivan, M. Functional Annotation of Hypothetical Proteins Derived from Suppressive Subtraction Hybridization (SSH) Analysis Shows NPR1 (Non-Pathogenesis Related)-Like Activity. Agronomy $2019,9,57$. [CrossRef]

132. Wang, Z.; Jia, C.; Li, J.; Huang, S.; Xu, B.; Jin, Z. Activation of salicylic acid metabolism and signal transduction can enhance resistance to Fusarium wilt in banana (Musa acuminata L. AAA group, cv. Cavendish). Funct. Integr. Genomics 2015, 15, 47-62. [CrossRef]

133. Cheng, Z.; Yu, X.; Li, S.; Wu, Q. Genome-wide transcriptome analysis and identification of benzothiadiazole-induced genes and pathways potentially associated with defense response in banana. BMC Genom. 2018, 19, 1-19. [CrossRef]

134. Thakker, J.N.; Patel, S.; Dhandhukia, P.C. Induction of defense-related enzymes in banana plants: Effect of live and dead pathogenic strain of Fusarium oxysporum f. sp. cubense. Isrn Biotechnol. 2013, 1-6. [CrossRef]

135. Nasir, N.; Dharma, A.; Habazar, T. The Chitinase Activity in Banana Seedling that Induce by Trichoderma spp as Resistance Responce to Fusarium Oxyporum f. sp. cubense. Int. J. Adv. Sci. Eng. Inf. Technol. 2016, 6, 356-360. [CrossRef]

136. Magdama, F.; Monserrate-Maggi, L.; Serrano, L.; Onofre, J.G.; Jiménez-Gasco, M.D.M. Genetic Diversity of Fusarium oxysporum f. sp. cubense, the Fusarium Wilt Pathogen of Banana, in Ecuador. Plants 2020, 9, 1133. [CrossRef]

137. Guo, L.; Han, L.; Yang, L.; Zeng, H.; Fan, D.; Zhu, Y.; Feng, Y.; Wang, G.; Peng, C.; Jiang, X.; et al. Genome and Transcriptome Analysis of the Fungal Pathogen Fusarium oxysporum f. sp. cubense Causing Banana Vascular Wilt Disease. PLoS ONE 2015, 10, e0117621. [CrossRef]

138. Staver, C.; Pemsl, D.E.; Scheerer, L.; Vicente, L.P.; Dita, M. Ex ante assessment of returns on research investments to address the impact of Fusarium wilt tropical race 4 on global banana production. Front. Plant. Sci. 2020, 11. [CrossRef]

139. Linbing, X.; Hu, Y.; Bingzhi, H.; Yuerong, W. Banana research and production in China. In Advancing Banana and Plantain RED in Asia and the Pacific; Molina, A.B., Xu, L.B., Roa, V.N., Van den Berghand, I., Borromeo, K.H., Eds.; International Network for the Improvement of Banana and Plantain (INIBAP): Brussels, Belgium, 2004; Volume 13, p. 51.

140. Simmonds, N.W. Evolution of Bananas; Longman: London, UK, 1962; pp. 5-25.

141. Bancroft, J. Report of the board appointed to enquire into the cause of disease affecting livestock and plants. Votes Proc. 1876, 3 , 1011-1038.

142. O’Neill, W.T.; Henderson, J.; Pattemore, J.A.; O’Dwyer, C.; Perry, S.; Beasley, D.R.; Tan, Y.P.; Smyth, A.L.; Goosem, C.H.; Thomson, K.M.; et al. Detection of Fusarium oxysporum f. sp. cubense tropical race 4 strain in northern Queensland. Australas. Plant. Dis. Notes 2016, 11, 33. [CrossRef]

143. Scheerer, L.; Pemsl, D.; Staver, C.; Dita, M.; Vicente, L.P. A Quantified Approach to Projecting Losses Caused by Fusarium Wilt Tropical Race 4; International Society for Horticultural Science: Brussels, Belgium, 2016; Volume 1196, pp. 211-218. [CrossRef]

144. D'Hont, A.; Denoeud, F.; Aury, J.M.; Baurens, F.C.; Carreel, F.; Garsmeur, O.; Noel, B.; Bocs, S.; Droc, G.; Rouard, M.; et al. The banana (Musa acuminata) genome and the evolution of monocotyledonous plants. Nature 2012, 488, 213-217. [CrossRef]

145. Raman, A.S.; White, K.I.; Ranganathan, R. Origins of allostery and evolvability in proteins: A case study. Cell 2016, 166, 468-480. [CrossRef]

146. Dong, H.; Ye, Y.; Guo, Y.; Li, H. Comparison transcriptome analysis revealed resistance differences of Cavendish bananas to Fusarium oxysporum $\mathrm{f}$. sp. cubense race1 and race 4. BMC Genom. 2020, 21, 122. [CrossRef]

147. Li, W.M.; Dita, M.; Rouard, M.; Wu, W.; Roux, N.; Xie, J.H.; Ge, X.J. Deep RNA-seq analysis reveals key responding aspects of wild banana relative resistance to Fusarium oxysporum f. sp. cubense tropical race 4. Funct. Integr. Genom. 2020, 20, 551-562. [CrossRef]

148. Chen, Y.C.; Wong, C.L.; Muzzi, F.; Vlaardingerbroek, I.; Kidd, B.N.; Schenk, P.M. Root defense analysis against Fusarium oxysporum reveals new regulators to confer resistance. Sci. Rep. 2014, 2014, 5584. [CrossRef]

149. Wang, Z.; Miao, H.; Liu, J.; Xu, B.; Yao, X.; Xu, C.; Zhang, J. Musa balbisiana genome reveals subgenome evolution and functional divergence. Nat. Plants 2019, 5, 810-821. [CrossRef]

150. Davey, M.W.; Gudimella, R.; Harikrishna, J.A.; Sin, L.W.; Khalid, N.; Keulemans, J. A draft Musa balbisiana genome sequence for molecular genetics in polyploid, inter-and intra-specific Musa hybrids. BMC Genom. 2013, 14, 683. [CrossRef] [PubMed] 
151. Wei, Y.; Liu, W.; Hu, W.; Liu, G.; Wu, C.; Liu, W.; Zeng, H.; He, Z.; Shi, H. Genome-wide analysis of autophagy-related genes in banana highlights MaATG8s in cell death and autophagy in immune response to Fusarium wilt. Plant. Cell Rep. 2017, 36, 1237-1250. [CrossRef]

152. Hwang, S.C.; Ko, W.H. Cavendish banana cultivars resistant to Fusarium wilt acquired through somaclonal variation in Taiwan. Plant. Dis. 2004, 88, 580-588. [CrossRef] [PubMed]

153. Ferreira, M.D.S.; Moura, É.R.D.; Lino, L.S.M.; Amorim, E.P.; Santos-Serejo, J.A.D.; Haddad, F. Selection of somaclonal variants of the cultivar 'Prata-Anã' for resistance to Fusarium oxysporum f. sp. cubense race 1. Rev. Bras. Frutic. 2020, 42. [CrossRef]

154. Ganapathi, T.R.; Higgs, N.S.; Balint-Kurti, P.J.; Arntzen, C.J.; May, G.D.; Van Eck, J.M. Agrobacterium-mediated transformation of embryogenic cell suspensions of the banana cultivar Rasthali (AAB). Plant. Cell Rep. 2001, 20, 157-162. [CrossRef]

155. Khanna, H.; Becker, D.; Kleidon, J.; Dale, J.L. Centrifugation assisted Agrobacterium tumefaciens-mediated transformation (CAAT) of embryogenic cell suspensions of banana (Musa spp. Cavendish AAA and Lady finger AAB). Mol. Breed. 2004, 14, 239-252. [CrossRef]

156. Damodaran, T.; Kumar, N.; Kavino, M. Breeding and evaluation of Musa hybrids resistant to Fusarium oxysporum f. sp. cubense race 1. Fruits 2009, 64, 3-12. [CrossRef]

157. Brown, A.; Tumuhimbise, R.; Amah, D.; Uwimana, B.; Nyine, M.; Mduma, H.; Talengera, D.; Karamura, D.; Kuriba, J.; Swennen, R. The genetic improvement of bananas and plantains (Musa spp.). In Genetic Improvement of Tropical Crops; Caligari, P.D.S., Ed.; Springer: Cham, Switzerland, 2017; pp. 219-240.

158. Nyine, M.; Uwimana, B.; Blavet, N.; Hřibová, E.; Vanrespaille, H.; Batte, M.; Akech, V.; Brown, A.; Lorenzen, J.; Swennen, R.; et al. Genomic prediction in a multiploid crop: Genotype by environment interaction and allele dosage effects on predictive ability in Banana. Plant. Genome 2018, 11. [CrossRef]

159. Dale, J.; Paul, J.Y.; Dugdale, B.; Harding, R. Modifying bananas: From transgenics to organics? Sustainability 2017, 9, 333. [CrossRef]

160. Ntui, V.O.; Tripathi, J.N.; Tripathi, L. Robust CRISPR/Cas9 mediated genome editing tool for banana and plantain (Musa spp.). Curr. Plant. Biol. 2019, 21, 100128. [CrossRef]

161. Tripathi, L.; Ntui, V.O.; Tripathi, J.N. Application of genetic modification and genome editing for developing climate-smart banana. Food Energy Secur. 2019, 8, e00168. [CrossRef]

162. Tripathi, J.N.; Ntui, V.O.; Ron, M.; Muiruri, S.K.; Britt, A.; Tripathi, L. CRISPR/Cas9 editing of endogenous banana streak virus in the B genome of Musa spp. overcomes a major challenge in banana breeding. Commun. Biol. 2020, 2, 1-11. [CrossRef]

163. Tripathi, L.; Ntui, V.O.; Tripathi, J.N. CRISPR/Cas9-based genome editing of banana for disease resistance. Curr. Opin. Plant Biol. 2020, 56, 118-126. [CrossRef]

164. Arora, S.; Steuernagel, B.; Gaurav, K.; Chandramohan, S.; Long, Y.; Matny, O.; Johnson, R.; Enk, J.; Periyannan, S.; Singh, N.; et al. Resistance gene cloning from a wild crop relative by sequence capture and association genetics. Nat. Biotechnol. $2019,37,139-143$. [CrossRef]

165. Jupe, F.; Witek, K.; Verweij, W.; Sliwka, J.; Pritchard, L.; Etherington, G.J.; Maclean, D.; Cock, P.J.; Leggett, R.M.; Bryan, G.J.; et al. Resistance gene enrichment sequencing (RenSeq) enables reannotation of the NB-LRR gene family from sequenced plant genomes and rapid mapping of resistance loci in segregating populations. Plant. J. 2013, 76, 530-544. [CrossRef] 